\title{
Regaining control of your emotions? Investigating the mechanisms underlying effects of cognitive control training for remitted depressed patients
}

\author{
Kristof Hoorelbeke*1, Nathan Van den Bergh*1, Rudi De Raedt ${ }^{1}$, Marieke Wichers ${ }^{2}$, Casper J. \\ Albers $^{3}$, \& Ernst H. W., Koster ${ }^{1}$ \\ ${ }^{1}$ Psychopathology and Affective Neuroscience (PAN) lab, Department of Experimental Clinical \\ and Health Psychology, Ghent University, Ghent, Belgium \\ ${ }^{2}$ University of Groningen, Department of Psychiatry, Interdisciplinary Center Psychopathology \\ and Emotion Regulation (ICPE), University Medical Center Groningen, The Netherlands \\ ${ }^{3}$ University of Groningen, Department of Psychometrics and Statistics
}

\section{Short Title: COGNITIVE CONTROL TRAINING AND EMOTION REGULATION DYNAMICS}

Keywords: cognitive control, cognitive remediation, cognitive training, network analysis, emotion regulation dynamics, depression

Note: Kristof Hoorelbeke and Nathan Van den Bergh contributed equally to the manuscript and share first authorship.

DISCLAIMER: This manuscript has been accepted for publication in Emotion. This document is the uncorrected word version (prior to editorial typesetting / proof print) 
*Corresponding Authors

Kristof Hoorelbeke, Nathan Van den Bergh

Department of Experimental Clinical and Health Psychology

Ghent University

Henri-Dunantlaan 2

Ghent, 9000, Belgium

Tel: +32 9.264.94.16

E-mail: kristof.hoorelbeke@ugent.be,nathan.vandenbergh@ugent.be 


\begin{abstract}
Studies suggest that cognitive control training shows potential as a preventive intervention for depression. At the same time, little is known regarding the mechanisms underlying effects of cognitive control training. Informed by theoretical frameworks of cognitive risk for recurrent depression (De Raedt \& Koster, 2010; Siegle et al., 2007), the current study sought to model direct effects of cognitive control training on the complex interplay between affect, emotion regulation, residual symptomatology, and resilience in a sample of remitted depressed patients $(n=92)$. Combining a four week experience sampling procedure with an experimental manipulation of cognitive control, we observed beneficial effects of cognitive control training on deployment of rumination. In addition, we obtained evidence for the causal involvement of cognitive control in efficacy of emotion regulation. In contrast to our expectations, cognitive control training did not exert immediate effects on residual symptomatology or resilience when compared to an active control condition, nor did cognitive control training impact the complex interplay between these variables. Overall, immediate effects of cognitive control training on functioning in daily life were limited.
\end{abstract}


Depressive episodes are characterized by marked perturbation of cognitive functions that frequently persist following remission from depression (e.g., Levens \& Gotlib, 2015). Provided that such cognitive impairments are associated with poor psychosocial functioning (Weightman et al., 2019), there has been a surge in studies exploring the potential of cognitive remediation strategies as preventive intervention for depression. Findings suggest that cognitive remediation may reduce depression vulnerability, as shown by beneficial effects of computerized cognitive training approaches on indicators of severity of depressive symptomatology, cognitive functioning (Motter et al., 2016; Launder et al., 2021), and use of maladaptive emotion regulation strategies such as rumination (Koster et al., 2017).

Specifically, cumulative evidence suggests that cognitive control deficits - among which difficulties in shifting, inhibition, and updating of information in working memory (Miyake et al., 2000) - form a risk factor for depression. That is, these executive functions are crucial for flexible adaptation of one's thoughts and behavior as a function of one's goals (Braver et al., 2002; Cohen, 2017). As such, cognitive control impairments have typically been linked to dysfunctional thought processes and use of maladaptive emotion regulation strategies (Joormann \& Vanderlind, 2014; Mor \& Daches, 2015), where cognitive control seems to be a necessary condition for adaptive and flexible emotion regulation (Pruessner et al., 2020). In this context, previous studies suggest that cognitive control is inversely related to the amount of previous depressive episodes (Vanderhasselt \& De Raedt, 2009) and number of hospitalizations (Harvey et al., 2004), placing one at-risk for recurrence of depression via use of maladaptive emotion regulation strategies (Demeyer et al., 2012).

Indeed, there is compelling evidence for the involvement of cognitive control in emotion regulation processes, via which cognitive control deficits may place one at risk for the development and maintenance of depressive symptoms (e.g., Hsu et al., 2015; Snyder \& Hankin, 2016). That is, 
experimental manipulation of cognitive control - using cognitive training procedures such as the adaptive Paced Auditory Serial Addition Task (adaptive PASAT; Siegle et al., 2007) or the adaptive n-back task (Jaeggi et al., 2008) - has shown to beneficially impact emotion regulation and depressive symptomatology (Beloe \& Derakhshan, 2019; Calkins et al., 2015; Iacoviello et al., 2014; Hoorelbeke \& Koster, 2017). For instance, in a sample of 23 patients suffering from major depressive disorder, Siegle and colleagues (2007) observed beneficial effects of a cognitive control training procedure consisting of six sessions of adaptive PASAT training, combined with Wells' attention training, on depressive rumination and severity of depressive symptomatology compared to a treatment as usual control group.

Importantly, in the context of depression vulnerability, beneficial effects of adaptive PASAT training have been repeatedly observed in healthy (e.g., Hoorelbeke, Koster, et al., 2016), at-risk (e.g., Calkins et al., 2015; Hoorelbeke, Koster, et al., 2015), and clinical samples (e.g., Brunoni et al., 2014; Siegle et al., 2014), suggesting that adaptive PASAT training shows potential as a preventive intervention for depression. At the same time, however, not all studies have consistently observed beneficial effects of adaptive PASAT training on indicators of depression vulnerability (e.g., Moshier et al., 2015; Moshier \& Otto, 2017; for a review, see Koster et al., 2017). Moreover, relatively little is known regarding the mechanisms via which adaptive PASAT training may impact risk for depression. Although several studies have focused on evaluating the effectiveness of cognitive control training procedures (in terms of establishing cognitive and emotional transfer effects), relatively few studies have focused on elucidating the mechanisms underlying effects of adaptive PASAT training.

In this context, influential conceptual frameworks assume that remediating cognitive control deficits may impact (risk for) depression via immediate effects on emotion regulation processes. It has been suggested that cognitive control training procedures such as the adaptive 
PASAT require recruitment of the dorsolateral prefrontal cortex while downregulating limbic interference (Siegle et al., 2007). On a behavioral level, the cognitive gains stemming from this may translate into more flexible and adaptive emotion regulation processes, among which less use of depressive rumination, via which cognitive control training may impact affective state and depressive symptoms (De Raedt \& Koster, 2010). For instance, cognitive control is considered key to foster disengagement from irrelevant repetitive negative thoughts, allowing to counter habitual ruminative response tendencies (Koster et al., 2011; Watkins \& Roberts, 2020).

In line with this, several studies suggest improvements at the level of dorsolateral prefrontal cortex functioning following cognitive control training (Cohen et al., 2016; Siegle et al., 2007). Furthermore, recent findings indicate that cognitive gains following cognitive control training in remitted depressed patients $(n=68)$ predict reduced rumination post-training, which further predicts beneficial effects on depressive symptomatology (Hoorelbeke \& Koster, 2017). However, these studies are limited in that they have typically relied on a limited number of assessments in highly controlled lab settings to evaluate effects of cognitive control training on emotion regulation and depressive symptomatology.

A different methodology, as well as a dynamic operationalization of emotion regulation, is necessary to shed light on the mechanisms underlying effects of cognitive control training in daily life. Interestingly, Experience Sampling Methodology (Larson \& Csikszentmihalyi, 1983) allows multiple assessments of mood and emotion regulation per day for a prolonged period, making it possible to investigate how cognitive control impacts emotion regulation dynamics. For instance, prospective findings suggest that cognitive control moderates the efficacy of emotion regulation in daily life (e.g., Pe, Raes, \& Kuppens, 2013; Pe, Raes, Koval, et al., 2013). As to date, one experimental study explored effects of cognitive control on emotion regulation dynamics in daily life, reporting effects of adaptive PASAT training compared to an active control condition on 
deployment of rumination ( $n=61$; Hoorelbeke, Koster, et al., 2016). However, this study only included a one-week experience sampling period following the cognitive control training procedure, and as such did not allow to model pre-post, and immediate effects of cognitive control training. Moreover, this study was conducted in a convenience sample, whereas most promising effects of cognitive control training have typically been observed in at-risk groups (e.g., remitted depressed patients) or clinical samples (e.g., patients suffering from major depressive disorder; Koster et al., 2017).

As such, a first aim of the current study is to model direct effects (i.e., effects emerging during and closely following the intervention period) of adaptive PASAT training on emotion regulation dynamics, and in particular, deployment and efficacy of emotion regulation, in remitted depressed patients. In this context, deployment refers to the extent to which being in a given affective state predicts future use of emotion regulation strategies. Efficacy, on the other hand, relates to changes in affect following the use of a given emotion regulation strategy (i.e., the impact of emotion regulation on affect; Hoorelbeke, Koster, et al., 2016).

In addition to previously observed beneficial effects of cognitive control training on emotion regulation and depressive symptomatology, studies using adaptive PASAT training as a preventive intervention for depression have also reported beneficial effects of experimental manipulation of cognitive control on broader indicators of functioning. For instance, in an experimental study in which at-risk undergraduate students (showing elevated trait rumination scores; $n=47$ ) were randomized over a cognitive control and an active control training condition consisting of an adaptive visual search task, Hoorelbeke, Koster and colleagues (2015) observed beneficial effects of adaptive PASAT training on reactivity to a lab stressor. In addition, in a recent double-blind randomized controlled trial evaluating effects of adaptive PASAT versus an active control training (consisting of a low load modification of the adaptive PASAT, a speed of response 
training) in remitted depressed patients $(n=68)$, Hoorelbeke and Koster (2017) observed beneficial effects on self-reported resilience and disability. In this context, resilience refers to the perceived ability to cope with adversity, including aspects such as inner strength and optimism (Wagnild \& Young, 1993; Wagnild, 2009), which has been closely linked to use of adaptive emotion regulation strategies such as positive appraisal (Kalisch et al. 2015).

Noteworthy, previous studies suggest immediate transfer of adaptive PASAT training on behavioral measures of cognitive functioning, among which indicators of task-specific cognitive transfer (as assessed using a non-adaptive version of the PASAT; Hoorelbeke \& Koster, 2017; Peckham \& Johnson, 2018; Vervaeke et al., 2020) and performance gains on untrained cognitive control tasks (e.g., antisaccade task or n-back task performance; Hoorelbeke, Koster, et al., 2016; Peckham \& Johnson, 2018; Segrave et al., 2014). In line with this, in a subsample consisting of six patients suffering from unipolar depression, Siegle and colleagues (2007) investigated effects of adaptive PASAT training on patterns of neural activity, reporting changes in neural activity at the level of the dorsolateral prefrontal cortex following training.

Together, these findings provide evidence for the validity of the adaptive PASAT as an experimental manipulation of cognitive control (Koster et al., 2017). In contrast to immediate performance gains on behavioral measures following cognitive control training, effects of adaptive PASAT training on self-reported cognitive complaints seem to develop over time. For instance, in the double-blind randomized controlled trial of Hoorelbeke and Koster (2017), remitted depressed patients which completed the adaptive PASAT training reported a marginal significant decrease in cognitive complaints from baseline to post-training, followed by a significant decrease in cognitive complaints from post-training to three months follow-up. This was not the case for the active control condition. In addition, compared to the control group remitted depressed patients reported increased resilience three months following adaptive PASAT training (Hoorelbeke \& Koster, 
2017). Furthermore, the long-term transfer effects of the current study suggest beneficial effects of cognitive control training on risk for recurrence of depression over a period of one year follow-up (Hoorelbeke et al., 2021).

Although existing conceptual frameworks suggest a key role of emotion regulation in obtaining such broad transfer effects (e.g., De Raedt \& Koster, 2010), as to date it remains to be tested how experimental manipulation of cognitive control may add to being resilient rather than vulnerable for the development of depressive symptoms. This reflects a strong need for mechanistic research exploring immediate effects of cognitive control training. In particular, following up on previous studies exploring the complex associations between these constructs following remission from depression (Hoorelbeke, Marchetti, et al., 2016; Hoorelbeke et al., 2019), it would be interesting to model how cognitive control training impacts the dynamic interplay between indicators of (mal)adaptive emotion regulation, such as rumination and positive appraisal, residual cognitive- and depressive complains, and resilience.

Interestingly, recent statistical developments, such as Vector AutoRegressive (VAR) network models, allow to model and visualize such level of complexity in a data-driven and intuitive manner (Borsboom \& Cramer, 2013; Bringmann et al., 2016). In particular, the combination of experience sampling and network analysis shows promise to increase our understanding of effects of interventions, as they allow to model how changes in one psychological construct in the model (referred to as 'nodes'; e.g., emotion regulation) may - via its unique associations with other nodes (referred to as 'edges') and positive feedback loops - cascade into a more profound transition of the system (Hofmann et al., 2016). For instance, recent studies using intensive time series data have relied on network analysis to model the impact of Imipramine and Mindfulness Based Cognitive Therapy on momentary mental states (Snippe et al., 2017). However, these highly innovative studies were limited to pre - post intervention comparisons. In order to 
model direct effects of cognitive control training, we propose a continuous assessment of emotion regulation, cognitive complaints, depressive symptomatology, and resilience throughout the intervention period. Here, we sought to model how cognitive control training impacts the temporal dynamics between each of these constructs. This can provide insights in the working mechanisms underlying previously reported beneficial effects of cognitive control training, illustrating how experimental manipulation of cognitive control impacts emotion regulation processes and how this potentially translates into fluctuations in residual depressive symptoms in daily life. For instance, modelling the impact of cognitive control training using temporal network models, which allow to visualize lagged predictive relations, depicting the extent to which activation of a given node at time $t$ uniquely predicts activation in other connected nodes at time $t+1$, including an autocorrelation for each of the nodes in the network. In addition, it would also be informative to model how cognitive control training affects patterns of simultaneous / co-occurring activity. Such patterns of activation are captured in contemporaneous networks, depicting the predictive relation between each of the variables within the same momentary assessment period (Epskamp et al., 2018).

\section{Current Study}

Given the role of cognitive control and emotion regulation in risk for recurrence of depression, in the current study we investigated direct effects of adaptive PASAT training in remitted depressed patients on emotion regulation processes, and how these relate to fluctuations in residual cognitive- and depressive complaints, as well as resilience. Building on recent methodological and statistical innovations, the current study set out to model the impact of cognitive control in daily life, as this is key to gain a mechanistic understanding of previously reported preventative effects of adaptive PASAT training in the literature (e.g., Hoorelbeke \& Koster, 2017), and how these effects may develop. 
For this purpose, remitted depressed patients participated in a four week experience sampling procedure. The first week provided baseline assessments of functioning. These assessments continued during weeks two and three which contained the experimental manipulation. In line with our previous studies (Hoorelbeke \& Koster, 2017; Hoorelbeke, Koster, et al., 2016), the experimental manipulation consisted of 10 sessions of adaptive PASAT training (cognitive control training) or a speed of response training task that was used as an active control condition. In previous work, we found that this procedure has effects on emotion regulation in remitted depressed individuals (Hoorelbeke \& Koster, 2017). Finally, the fourth week of experience sampling provided assessments of post-training functioning in daily life. This project was preregistered on Open Science Framework (osf.io/g2k4w). To increase our understanding of the working mechanisms underlying adaptive PASAT training, we aimed to:

(1) Evaluate immediate effects of cognitive control training on emotion regulation dynamics. In line with Hoorelbeke, Koster, et al. (2016) we aimed to model effects of adaptive PASAT training on deployment and efficacy of rumination and positive appraisal in daily life;

(2) Evaluate immediate effects of cognitive control training in terms of self-reported levels of (residual) cognitive and depressive complaints in daily life, as well as self-reported resilience;

(3) Explore the mechanisms underlying emotional transfer effects of cognitive control training, using network analysis to model immediate effects of adaptive PASAT training on the dynamics between emotion regulation, residual symptomatology (cognitive complaints, depressive symptomatology), and self-reported resilience in daily life.

\section{Method}




\section{Design}

This manuscript reports the short-term transfer effects of a preregistered single-blind randomized controlled trial (osf.io/g2k4w) aimed to evaluate effects of adaptive PASAT training for remitted depressed patients in daily life. The study consisted of: a screening phase, baseline clinical assessment, and an experience sampling procedure of four weeks in the midst of which participants engaged in the training procedure (week 1: baseline, weeks 2 - 3: experimental manipulation [cognitive control training / active control condition], week 4: post-training). In addition, long-term effects of the intervention were monitored weekly over a period of one year, which concluded with a follow-up assessment of functioning and recurrence. The current manuscript reports on the immediate effects of adaptive PASAT training in daily life, which is limited to the findings stemming from the four weeks of experience sampling (cf. osf.io/g2k4w). Participants were randomized over the training conditions using RandList software (randomisation.eu). They were blind to the training conditions used.

\section{Participants}

We used advertisements on social media and in local newspapers to recruit patients with a history of depression. Baseline assessment was conducted at Ghent University. Participants were allowed to enter the experiment if following criteria were met: (a) the participants were aged 2365 at the time of the baseline assessment, (b) participants reported a history of depression, and (c) participants were in possession of a smartphone with a data plan. In addition, (d) participants did not meet criteria for an ongoing depressive episode, nor reported current substance abuse or presence of a psychotic disorder. Based on power analyses conducted for our previous randomized controlled trial in which emotional transfer effects of adaptive PASAT training were modeled in remitted depressed patients (Hoorelbeke, Faelens, et al., 2015), our aim was to have a minimum of 
68 formerly depressed patients completing the experimental manipulation to be included in the analysis of short- and long-term effects of cognitive control training. Given the intensive study design, we expected significant drop-out. For this purpose, we oversampled. Enrollment of participants took place between March and July 2017. The study was approved by the local ethics committee and written informed consent was obtained for all participants.

\section{Materials}

\section{Screening Procedure}

Participants were screened for eligibility using an in time separated two-step screening procedure. Following an initial telephone screening containing the relevant modules of the MiniInternational Neuropsychiatric Interview (MINI; Van Vliet \& De Beurs, 2007), potential eligible participants were invited for a second screening at the Faculty of Psychology and Educational Science of Ghent University. During this visit, the Mood Disorders module of the MINI was (re)administered by a licensed clinical psychologist. The MINI is a structured clinical interview based on the Diagnostic and Statistical Manual of Mental Disorders, which allows to establish presence of a lifetime history of depression in absence of a current depressive episode.

\section{Baseline Questionnaires}

Several questionnaires were administered at baseline and were only reassessed at one year follow-up. As such, these measures could not be used to evaluate immediate emotional transfer following cognitive control training and are only used in the current manuscript to evaluate whether the randomization procedure was effective. We assessed residual depressive symptomatology using the Beck Depression Inventory (BDI-II-NL, range: $0-63$, Cronbach's $\alpha=.91$; Van der Does, 2002) and the Remission of Depression Questionnaire (RDQ, range: $0-82$, Cronbach's $\alpha=.78$; 
Peeters et al., 2013; Zimmerman et al., 2013). In addition, we assessed trait Positive (Cronbach's $\alpha=.89$ ) and Negative Affect (Cronbach's $\alpha=.80$ ) using the Positive and Negative Affect Scales (PANAS-NL, range 10 - 50; Engelen et al., 2006). We included several indicators of emotion regulation, among which the Ruminative Response Scale (RRS, total score: range $22-88$, Cronbach's $\alpha=.91$; subscale Brooding [depressive rumination]: range 5 -20 , Cronbach's $\alpha=.77$; subscale Reflection: range $5-20$, Cronbach's $\alpha=.72$; Treynor et al., 2003) and the Cognitive Emotion Regulation Questionnaire (CERQ, range adaptive emotion regulation: 20 - 100, Cronbach's $\alpha=.92$, range maladaptive emotion regulation: $16-80$, Cronbach's $\alpha=.80$; Garnefski et al., 2001). Cognitive dysfunctioning was assessed using the Behavior Rating Inventory of Executive Function Adult Version (BRIEF-A, Global Executive scale, range: 70 - 210, Cronbach's $\alpha=.94$; Scholte \& Noens, 2011), whereas resilience was assessed using the Resilience Scale (RSNL, range: $25-100$, Cronbach's $\alpha=.87$; Portzky, 2008). Finally, following the psycho-education section regarding the training procedure and experience sampling phase, which was part of the baseline assessment, participants completed the Credibility/Expectancy Questionnaire (CEQ, Credibility: Cronbach's $\alpha=.69$, Expectancy: Cronbach's $\alpha=.84$; Devilly \& Borkovec, 2000), allowing to test for motivational differences between both training conditions prior to the experience sampling phase and experimental manipulation.

\section{Baseline Cognitive Task Performance}

In addition to the BRIEF-A, participants completed a non-adaptive version of the PASAT (Gronwall, 1977) at baseline, offering an indicator of cognitive control ability prior to the experimental manipulation. The non-adaptive PASAT uses auditory stimuli, ranging from one to nine. During this task, participants are presented with a stream of digits and have to continuously respond to the sum of the two most recently heard digits. During this task the inter-trial interval 
(ITI) is fixed (non-adaptive), allowing to measure cognitive control in a standardized manner. For this purpose, in line with previous cognitive control training studies (e.g., Hoorelbeke \& Koster, 2017), the non-adaptive PASAT consisted of three blocks, containing 60 test trials each. The intertrial interval (ITI) for the first block was $3000 \mathrm{~ms}$. The ITIs used during the second and third block, were 2000 and $1500 \mathrm{~ms}$ respectively. Before starting the test trials of the first block, participants completed ten practice trials, allowing participants to familiarize with the task.

\section{Experimental Manipulation}

Participants were randomized over two training conditions. In the cognitive control training condition participants were asked to complete ten training sessions based on an adaptive version of the PASAT (Siegle et al., 2007). During this task, participants are presented with a stream of digits (ranging from $1-9$ ) and have to continuously respond to the sum of the last two heard digits. The adaptive PASAT differs from the non-adaptive PASAT in that task difficulty is continuously adjusted based on one's performance. Each training session started with an ITI of 3000 ms. Every four consecutive correct/incorrect responses, the ITI decreased/increased with $100 \mathrm{ms,} \mathrm{adaptively}$ tailoring task difficulty to one's level of performance during the session. Each session started with practice trials and consisted of 400 training trials.

In contrast, participants in the active control condition were presented with an adaptive speed of response task, which was a modification of the adaptive PASAT task (Hoorelbeke, Koster, et al., 2016). During this task, participants are also confronted with a continuous stream of digits (range: 1 -18). However, instead of responding to the sum of the two most recently heard digits, participants were instructed to respond to the last heard digit as fast as possible. In line with the adaptive PASAT training, each training session of the active control condition started with an ITI 
of $3000 \mathrm{~ms}$, which decreased/increased with $100 \mathrm{~ms}$ every four consecutive correct/incorrect responses. This offers a low cognitive load version of the adaptive PASAT (i.e., during the adaptive PASAT one needs to continuously update information in working memory, while inhibiting task irrelevant information), where the adaptive nature of the active control task allows to control for motivational effects of undergoing training as well as more general attentional processes that are targeted by both training tasks. That is, previous studies indicate that the active control training and adaptive PASAT training procedure do not differ in the extent to which both training tasks are considered a credible intervention for participants, nor in terms of expectancies concerning potential treatment gains (Hoorelbeke \& Koster, 2017; Van den Bergh et al., 2020; Vervaeke et al., 2021). In addition, previous studies suggest user engagement to be equally high in both training tasks in remitted depressed patients (Vervaeke et al., 2021).

In both conditions, participants were instructed to complete ten training sessions over a period of two weeks (weeks two and three following the baseline assessment at the lab). Both groups received standardized psycho-education regarding the training procedure in order to foster task engagement (Siegle et al., 2014; cf. Hoorelbeke, Faelens, et al., 2015).

\section{Experience Sampling}

Participants completed four weeks (28 days) of experience sampling, during which they received six signals a day between 9 AM and 9 PM using a time-stratified assessment schedule. As such, each assessment day was divided into six blocks of two hours. Within each block, signals were sent at random moments, with the exception that the interval between two successive signals should keep a minimum of 30 minutes in order to avoid overlapping assessments. That is, participants were asked to respond to each of these signals as soon as possible, considering that 
signals timed-out after 30 minutes. In addition, if no response was given within the first 15 minutes, a reminder signal was sent. Using SurveySignal (Hofmann \& Patel, 2015), signals were sent as text messages each of which contained a unique link directing the participant to the online questionnaire on a LimeSurvey platform.

The online questionnaire that was repeatedly assessed in daily life consisted of 13 items: (a) In line with Hoorelbeke, Koster, et al. (2016), six items were used to assess the current affective state of the participants ('energetic', 'happy', 'satisfied', 'angry', 'tense', and 'depressed'). A compound score for affect was computed, where higher scores reflect a more positive / less negative affective state; (b) The occurrence of (un)pleasant events was monitored using one item ("Reflect upon the most influential event that has occurred since the previous signal. How (un)pleasant was this?"), allowing to test whether both groups would differ in terms of occurrence of life events; (c) Three items assessed emotion regulation processes, two of which were adopted from Moberly and Watkins (2008) to measure the occurrence of rumination in daily life ('focused on feelings', 'focused on problems'). Participants were instructed that these items assess the extent to which participants experience repetitive negative thinking regarding either feelings or problems. In line with Hoorelbeke, Koster, et al. (2016), a third emotion regulation item was used to assess positive appraisal ('focus on a positive meaning'), defined as the extent to which participants cognitively (re)appraised events in a positive manner. (d) Participants also rated the extent to which they considered themselves able to effectively cope with adversity in daily life, referred to as 'resilience' ('felt resilient'). (e) Finally, two items assessed occurrence of residual complaints, among which one item assessed the extent to which participants 'experienced cognitive complaints' and one item assessed the extent to which participants 'experienced depressive symptomatology'. Participants were instructed that the cognitive complaints item referred to the extent to which they 
experienced difficulties in daily life related to impaired working memory or broader executive functioning (e.g., attention). In contrast, the depression item referred to main depressive features based on the Diagnostic and Statistical Manual of Mental Disorders (i.e., being in a state of sustained negative affect or experiencing anhedonic features such as loss of pleasure / interest in daily life).

The affective items were presented first in random order, followed by the item regarding occurrence of (un)pleasant events. Participants then continued with the emotion regulation-, resilience-, and residual symptomatology items which were presented in random order. All items were rated on a scale from 1 to 100 , where participants were instructed to enter the number that corresponded to their state. With the exception of the item regarding occurrence of (un)pleasant events, for each item 1 corresponded to "not at all" and 100 to "very much". For the item pertaining occurrence of (un)pleasant events, 1 corresponded to "very unpleasant" and 100 to "very pleasant". Participants rated all items with regards to the interval between the current signal and the previous signal the participant responded to, except for the indicators of positive and negative affect. In addition, the first signal of the experience sampling period related to the period that had extended since waking up.

Participants received written and oral instructions regarding the content of the items during the baseline lab visit. In addition, to familiarize participants with these items, participants completed the items for the first time during the baseline lab visit. The psychometric properties of these items have been investigated during the follow-up phase of this project, reported in Hoorelbeke et al. (2021), where - using a different timescale - the items were validated against standardized measures for emotion regulation, cognitive complaints, depressive symptomatology, 
and resilience (for a detailed report on this, please see Hoorelbeke et al., 2021 supplemental material).

\section{Procedure}

Candidate participants completed an in time separated two-phased screening procedure using a structured clinical interview. After providing informed consent, eligible remitted depressed patients were randomized to the cognitive control training or active control condition and completed a baseline assessment of functioning at Ghent University containing several questionnaires and a behavioral measure for cognitive control (i.e., the non-adaptive PASAT). In addition, participants received standardized psycho-education regarding the experience sampling phase and the cognitive training procedure, after which they filled out a questionnaire to evaluate the motivation of participants to engage in the training study (credibility and expectancy). At the end of the baseline assessment, participants' smartphones were registered in SurveySignal. The experience sampling phase commenced the day following the baseline lab assessment and lasted for a period of four weeks, containing six assessments a day between 9 AM and 9 PM. The first week offered a baseline assessment of daily life functioning. Weeks two and three contained the experimental manipulation. That is, during week two and three of the experience sampling phase, participants were instructed to complete the online training procedure. For this purpose, participants received an automated daily reminder signal via text message to prompt them to conduct the training. The fourth week of the experience sampling phase offered a post-training assessment of effects of cognitive control training in daily life. Together, this experience sampling procedure allows to evaluate immediate effects of cognitive control training in daily life. Albeit outside the scope of the current manuscript, it should be noted that participants were further monitored on a weekly basis over a period of one year following training using online assessments. 
At the end of this follow-up procedure, participants returned to the lab for a reassessment of cognitive and emotional functioning, in addition to assessment of recurrence of depression over the past year. At the end of this session, participants were debriefed and received financial reimbursement for participation to the study. The long-term effects of cognitive control training are reported elsewhere (Hoorelbeke et al., 2021).

\section{Data-analysis}

Data-analysis was conducted in $\mathrm{R}$ (version 3.6.1) where we excluded all participants that completed less than $50 \%$ of the assessments over the 28 day experience sampling period.

\section{Training Task Progress}

Progress on the training tasks will be modeled using median ITI scores per session. In line with prior studies (Hoorelbeke, Koster, et al., 2016), given that both tasks differ, we will conduct two separate Repeated Measures ANOVAs to assure that training task performance significantly increased over time (10 sessions).

\section{Emotion Regulation Dynamics}

To address our first research question, we modeled effects of cognitive control training on deployment and efficacy of emotion regulation in daily life. For this purpose, we relied on Hierarchical Linear Models. For each of the Hierarchical Linear Models presented in this manuscript, we used an autoregressive AR(1) structure to account for the temporal correlation within an individual. Where necessary for the lagged variables, subject centering was used. These analyses were conducted in $\mathrm{R}$, using the nlme package (for version info, see supplemental material; Pinheiro et al., 2019). We relied on marginal $R^{2}\left(R_{m}^{2}\right.$; Nakagawa \& Schielzeth, 2013), estimated using the $r 2 \mathrm{glmm}$ package, as an indicator of effect-size. $R_{m}^{2}$ reflects the explained variance of the fixed effects. Follow-up simple slope analyses to facilitate interpretation of significant interaction 
effects were conducted using the reghelper package (Hughes, 2020) using \pm 2 SDs as this roughly corresponds with a $95 \%$ confidence interval.

Deployment of Emotion Regulation. We first modeled effects of cognitive control training on deployment of emotion regulation using Hierarchical Linear Models. Separate analyses were conducted for every Emotion regulation strategy (Rumination / Positive Appraisal). At level 1 we modeled how use of Emotion regulation at time $t$ for individual $\mathrm{j}\left(\mathrm{Y}_{\mathrm{t}, \mathrm{j}}\right)$, was a function of Time $\left(\mathrm{X}_{\mathrm{t}, \mathrm{j}}\right)$ and Affect at time $\mathrm{t}-1\left(\mathrm{Z}_{\mathrm{t}-\mathrm{i}, \mathrm{j}}\right)$. In addition, we added an interaction term to test whether the relation between Affect and Emotion regulation changed throughout the four week experience sampling period $\left(\mathrm{X}_{\mathrm{t}, \mathrm{j}} \times \mathrm{Z}_{\mathrm{t}-1, \mathrm{j}}\right)$.

$$
Y_{t, j}=\beta_{0 j}+\beta_{1 j} X_{t, j}+\beta_{2 j} Z_{t-1, j}+\beta_{3 j} X_{t, j} \times Z_{t-1, j}+e_{i j}
$$

At level 2, we modeled how the intercept $\left(\beta_{0 \mathrm{j}}\right)$ and the slopes of Time $\left(\beta_{1 \mathrm{j}}\right)$, Affect $\left(\beta_{2 \mathrm{j}}\right)$, and the corresponding interaction term $\left(\beta_{3 \mathrm{j}}\right)$ were a function of Group (cognitive control or active control condition). Based on the Bayesian Information Criterion parameter, optimal model fit was obtained when allowing for a random intercept $\left(b_{0 j}\right)$ and random slopes for Time and Affect $\left(b_{1 j}\right.$ and $b_{2 j}$ ). That is, for analysis of effects of cognitive control training on deployment of Emotion regulation in daily life, this model was preferred over a model that included an additional random slope for the interaction term $\left(b_{3 j}\right)$.

$$
\begin{aligned}
& \beta_{0 j}=\gamma_{00}+\gamma_{01} \operatorname{Group}_{j}+b_{0 j} \\
& \beta_{1 j}=\gamma_{10}+\gamma_{11} \text { Group }_{j}+b_{1 j} \\
& \beta_{2 j}=\gamma_{20}+\gamma_{21} \text { Group }_{j}+b_{2 j} \\
& \beta_{3 j}=\gamma_{30}+\gamma_{31} \text { Group }_{j}
\end{aligned}
$$


Efficacy of Emotion Regulation. In order to evaluate effects of cognitive control training on efficacy of emotion regulation, we again fitted separate Hierarchical Linear Models for each Emotion regulation strategy. In particular, at level 1 we modeled the impact of use of a given Emotion regulation strategy in the period preceding the assessment (rated at time $t ; \mathrm{Z}_{\mathrm{t}, \mathrm{j}}$ ) on Affect at time $\mathrm{t}\left(\mathrm{Y}_{\mathrm{t}, \mathrm{j}}\right)$, while controlling for Affect at time $\mathrm{t}-1\left(\mathrm{Y}_{\mathrm{t}-1, \mathrm{j}}\right)$. In addition, we modeled the effect of Time $\left(\mathrm{X}_{\mathrm{t}, \mathrm{j}}\right)$, and included an interaction term to allow changes in the slope between Emotion regulation and Affect over Time $\left(\mathrm{X}_{\mathrm{t}, \mathrm{j}} \times \mathrm{Z}_{\mathrm{t}, \mathrm{j}}\right)$ :

$$
Y_{t, j}=\beta_{0 j}+\beta_{1 j} X_{t, j}+\beta_{2 j} Z_{t, j}+\beta_{3 j} Y_{t-1, j}+\beta_{4 j} X_{t, j} \times Z_{t, j}+e_{i j}
$$

At level 2, we modeled the intercept $\left(\beta_{0 \mathrm{j}}\right)$ and the slopes of Time $\left(\beta_{1 \mathrm{j}}\right)$, Emotion regulation $\left(\beta_{2 \mathrm{j}}\right)$, and the corresponding interaction term $\left(\beta_{4 \mathrm{j}}\right)$ as a function of Group. Again relying on the Bayesian Information Criterion values to optimize model fit, we allowed a random intercept $\left(\mathrm{b}_{0 \mathrm{j}}\right)$ and random slopes for Time $\left(b_{1 j}\right)$, Emotion regulation $\left(b_{2 j}\right)$ and the interaction between Time and Emotion regulation $\left(b_{4 j}\right)$.

$$
\begin{aligned}
& \beta_{0 j}=\gamma_{00}+\gamma_{01} \operatorname{Group}_{j}+b_{0 j} \\
& \beta_{1 j}=\gamma_{10}+\gamma_{11} \text { Group }_{j}+b_{1 j} \\
& \beta_{2 j}=\gamma_{20}+\gamma_{21} \text { Group }_{j}+b_{2 j} \\
& \beta_{3 j}=\gamma_{30} \\
& \beta_{4 j}=\gamma_{40}+\gamma_{41} \text { Group }_{j}+b_{4 j}
\end{aligned}
$$

\section{Direct Effects of Cognitive Control Training on Cognitive Complaints, Depressive}

\section{Symptomatology and Resilience}

We continued using Hierarchical Linear Modeling to examine immediate effects of the experimental manipulation on fluctuations in Cognitive complaints, residual Depressive 
symptomatology, and self-reported Resilience in daily life. For each of these dependent measures $\left(\mathrm{Y}_{\mathrm{t}, \mathrm{j}}\right)$, we modeled the effect of Time $\left(\mathrm{X}_{\mathrm{t}, \mathrm{j}}\right)$ at level 1:

$$
Y_{t, j}=\beta_{0 j}+\beta_{1 j} X_{t, j}+e_{i j}
$$

The effect of Group on the slope of $X_{t, j}$ was modeled at level 2. We allowed random effects for the intercept and slope of $\mathrm{X}_{\mathrm{t}, \mathrm{j}}$.

$$
\begin{aligned}
& \beta_{0 j}=\gamma_{00}+\gamma_{01} \operatorname{Group}_{j}+b_{0 j} \\
& \beta_{1 j}=\gamma_{10}+\gamma_{11} \text { Group }_{j}+b_{1 j}
\end{aligned}
$$

\section{Network Analysis}

In order to address our third research question, we then continued with network analyses. We modeled effects of training on the dynamic interplay between emotion regulation (Positive Appraisal, Rumination), Cognitive complaints, Depressive symptomatology, and self-reported Resilience. For this purpose, responses to assessments during the experience sampling phase were categorized as belonging to the pre-training-, training-, or post-training phase based on individual training completion levels. That is, all responses made prior to completion of the first training session were categorized as belonging to the 'pre-training phase' (unique observations: active control condition: $n=1621$, cognitive control training: $n=1968)$. All signals following the last completed training session for a given subject were categorized as 'post-training' assessments (unique observations: active control condition: $n=1432$, cognitive control training: $n=1641$ ). All intermediate responses were used to model direct effects of cognitive training and were categorized as belonging to the 'training phase' (unique response: active control condition: $n=1868$, cognitive control training: $n=2338$ ). 
For each of these subsets, a two-step multilevel Vector AutoRegressive (VAR) approach ( $m l V A R$ package; Epskamp et al., 2017) was used to compute network models for the cognitive control training and active control condition. As a first step, this procedure models the relation between a given variable at time $t$ and all other subject centered variables at time $t-1$. Each of these models include an autocorrelation for the dependent variable. This allows computation of a temporal network, depicting how activation of a given node at time $\mathrm{t}$ predicts activation of connected nodes at time $t+1$. As a second step, using multilevel regression models, residuals of one variable at time $\mathrm{t}$ are predicted by all other variables at time $\mathrm{t}$. This allows computation of a contemporaneous network, which reflects patterns of co-occurring activity (i.e., patterns of activation of nodes within the same momentary assessment). For a more detailed description of the two-step multilevel VAR procedure implemented by $m l V A R$, please see Epskamp, Waldorp, et al. (2018).

The corresponding network models were plotted with the qgraph package (Epskamp et al., 2012), using the estimated coefficients as input. For the contemporaneous network, we implemented the OR-rule, meaning that edges between two given nodes were included in the model if at least one of both multivariate regressions was significant. In the corresponding network models, positive relations were depicted by blue edges, whereas negative relations were depicted by dashed red edges. Edge thickness reflects strength of connectivity. In the temporal networks, the arrows indicate the direction of the observed relations.

To examine the impact of the experimental manipulation on the obtained network models, we first computed network density, the weighted sum of all (absolute) obtained coefficients, after which we examined effects of the intervention on the obtained network structure(s). For this purpose, we calculated the Structural Hamming Distance for each of the obtained network models 
in the cognitive control training and active control condition, comparing them to their corresponding baseline model (within group). Structural Hamming Distance refers to the amount of differences between two given network models (i.e., insertions, omissions, and inversions of edges). As such, a small value suggests that the network models under comparison demonstrate highly similar network structures (Acid \& de Campos, 2003). To examine effects of the intervention on a single edge level, we then used two-group Z-tests (temporal networks) or Fisher Z-tests (contemporaneous networks) to test whether edges differed significantly between network models. In particular, for each of the groups, we tested whether the edges of the network models obtained during the training and post-training phase differed significantly from edges obtained in the corresponding baseline network model. We used Bonferroni correction to control for multiple comparisons. A temporal network model containing five nodes allows for a maximum of 25 edges (one incoming and one outgoing edge between each of the nodes, and an autocorrelation), whereas contemporaneous network models with five nodes allow for a maximum of 10 edges. Within each set of comparisons of network models at a single edge level, we corrected for this number of tests of significant edge differences between two given networks. As such, in order to be deemed statistically significant, for within- and between-subject comparisons of two given network models, p-values should be below .002 or .005 for temporal or contemporaneous networks respectively. Using the same sequence of analyses (Structural Hamming Distance, two-group Z-tests, Fisher Ztests), we then proceeded testing whether the obtained structures in the baseline-, training-, and post-training networks differed between both training conditions, again controlling for multiple comparisons.

\section{Results}




\section{Group Characteristics}

92 remitted depressed patients were randomized over the cognitive control training or active control condition (see Figure 1 for the CONSORT patient flow diagram). No significant group differences emerged for demographical variables, use of medication, or history of depression (Table 1; all $t \mathrm{~s}<.50$, all $\chi^{2} \mathrm{~s}<0.88$, for Fisher's Exact tests all $p \mathrm{~s}>.19$ ). In addition, both groups did not significantly differ on the baseline questionnaires or the cognitive assessment task (Table 1 ; all $t$ s $<1.96)$. Within the cognitive control training condition, 42 out of 47 participants completed the recommended amount of ten training sessions. This was the case for 38 out of 45 participants in the active control condition (Figure 1). No differences emerged in number of completed training sessions between conditions $(t(90)=0.05, p=.96)$. On average, participants in the cognitive control training condition and active control training condition completed $9.57(S D=2.21)$ and $9.60(S D$ $=3.07)$ training sessions respectively. Eight participants responded to $<50 \%$ of the assessments during the experience sampling phase and were excluded from these analyses ${ }^{1}$ (among which two participants who could not start the experience sampling procedure due to technical issues), resulting in a final sample of $n=84$ (ratio cognitive control training : active control condition $=46$ : 38) and a total of 10868 unique assessments. Both groups did not significantly differ in terms of response rates to the experience sampling procedure (cognitive control training: $M=76.95, S D=$ 12.55; active control condition: $M=77.27, S D=11.88 ; t(82)=0.12, p=.91$ ), nor did they differ in terms of response latency (in minutes; cognitive control training: $M=8.45, S D=2.07$; active control condition: $M=8.17, S D=1.79 ; t(82)=0.67, p=.51)$. Individuals showed considerable variability in how they responded to the repeated assessments over time, justifying the use of

\footnotetext{
${ }^{1}$ Participants who were excluded from these analyses based on response rates to the experience sampling procedure $<50 \%$ did not significantly differ from the other participants on any of the baseline measures, including self-reported expectancy and credibility of the training procedure (all $t s<1.88$ ).
} 
Hierarchical Linear Models with random intercept and slope(s). That is, the intra-class correlations for Rumination, Positive appraisal, Cognitive complaints, Depressive symptomatology, Resilience, and Affect were $.54, .58, .49, .44, .59$, and .51 respectively. Importantly, the occurrence of stressful life events throughout the experience sampling procedure did not differ between both conditions $(p=.35)$.

\section{Progress on the Training Task}

Both groups demonstrated a significant and large improvement in training task performance over Time (cognitive control training: $F(9,33)=20.18, p<.001, \eta_{p}^{2}=.85$; active control condition: $\left.F(9,29)=4.10, p<.01, \eta_{p}^{2}=.56\right)$. Group means for the median ITI scores per session are reported in supplemental Table 1, where a decrease in ITI is indicative for improvements on the training task.

\section{Effects of Cognitive Control Training on Emotion Regulations Dynamics \\ Deployment of Emotion Regulation}

Results from analysis of effects of cognitive control training on deployment of emotion regulation are presented in the upper half of Table 2. We first explored the relation between Affect and Rumination. Being in a better mood (i.e., higher scores on Affect) predicted lower subsequent

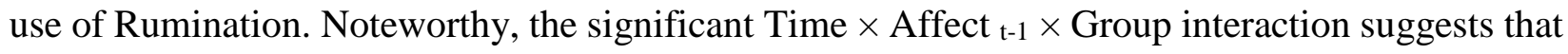
the experimental procedure affected deployment of Rumination in daily life (Figure 2).

We continued with follow-up simple slope analyses to facilitate interpretation of the significant three-way interaction effect. These analyses suggest that the experimental manipulation did not impact use of Rumination when being in a relatively negative Affective state. That is, for both conditions the extent to which being in a relatively bad Affective state (i.e., -2 SD below the 
subject centered mean for Affect) was predictive for use of Rumination, did not change over time ( $p$ s > .37). Instead, as time progressed the protective effect of being in a more positive Affective state (+2 SD) increased in the cognitive control training condition, predicting lower subsequent use of Rumination over Time $(\gamma=-0.07, S E=0.02, p<.001)$. Importantly, this seemed to be specific for the cognitive control training condition (active control condition: $\gamma=-0.01, S E=0.02, p=.66$ ). Similarly, we observed a tendency for a decrease in use of Rumination at mean levels of Affect in the cognitive control training condition $(\gamma=-0.03, S E=0.02, p=.055)$, whereas deployment of Rumination remained stable over time at mean levels of Affect in the active control condition $(\gamma=$ $-0.01, S E=0.02, p=.44$; Figure 2).

The results pertaining deployment of Positive Appraisal suggest that participants mostly engaged in this emotion regulation strategy when being in a more positive Affective state. In addition, we observed a significant Time $\times$ Affect $t-1$ interaction, suggesting that throughout the assessment period deployment of Positive Appraisal became more contingent on Affective state. However, this did not differ between both conditions. That is, the cognitive control training procedure did not significantly impact deployment of Positive Appraisal in daily life, as shown by the non-significant Time $\times$ Affect $_{\mathrm{t}-1} \times$ Group interaction term $(\mathrm{cf}$. Table 2).

\section{Efficacy of Emotion Regulation}

Results from the analyses pertaining efficacy of emotion regulation are presented in the lower half of Table 2. We first modeled effects of Rumination on Affect. Use of Rumination negatively impacted participants' Affective state. In addition, we observed a general improvement in Affect over time. These analyses also revealed several significant interactions, among which the Time $\times$ Rumination $\times$ Group interaction suggesting that the experimental manipulation impacted effectiveness of emotion regulation (Figure 3). 
Follow-up simple slope tests suggest that for the active control condition, being in a moderate (mean level: $\gamma=0.02, S E=0.01, p=.006)$ or high ruminative state (+2 SD: $\gamma=0.05, S E$ $=0.01, p<.001)$ had a less negative impact on affect over time. In contrast, we observed a tendency for the cognitive control training condition to report more negative affect over time when being in a high ruminative state $(\gamma=-0.02, S E=0.01, p=.08)$. For both conditions, the relation between being in a low ruminative state and Affect did not change over time (all other $p s>.15$ ). In order to gain a better understanding of this unexpected pattern of results, we used simple slope tests to model the predictive relation between Rumination and Affect during the mid of the baseline(signal 22) and post-training week (signal 148). For both the active control and cognitive control training condition we observed a negative impact of Rumination on Affect during the baseline (cognitive control training: $\gamma=-0.25, S E=0.02, p<.001$; active control condition: $\gamma=-0.27, S E=$ $0.02, p<.001$ ) and post-training experience sampling cycle (cognitive control training: $\gamma=-0.33$, $S E=0.04, p<.001$; active control condition: $\gamma=-0.13, S E=0.04, p=.003)$. However, in line with the findings presented above, the strength of the coefficients increased from baseline to posttraining in the cognitive control training condition, whereas the opposite pattern was observed in the active control condition. These findings indicate that, relative to the active control condition, over time cognitive control training increased the impact of Rumination on Affect.

For the analysis of Positive Appraisal, the Time $\times$ Positive Appraisal $\times$ Group interaction was non-significant, suggesting no effect of the experimental manipulation on efficacy of Positive Appraisal. That is, we only observed significant main effects of Time, use of Positive Appraisal, and prior Affective state. Overall, use of Positive Appraisal increased over time, with Positive Appraisal predicting improvements in Affect while controlling for prior Affective state.

\section{Immediate Effects of Cognitive Control Training on Residual Complaints and Resilience}


We observed a general decrease in Cognitive complaints throughout the four week experience sampling period $\left(\gamma=-0.06, t(10782)=3.60, p<.001, R_{m}^{2}=.006\right)$. However, no significant main effect of Group $\left(\gamma=-0.98, t(82)=0.26, p=.80, R_{m}^{2}<.001\right)$ or Time $\times$ Group interaction effect was observed $\left(\gamma=0.003, t(10782)=0.12, p=.91, R_{m}^{2}<.001\right)$.

Similarly, cognitive control training yielded no immediate effects on severity of Depressive symptoms in daily life. That is, the Time $\times$ Group interaction effect did not reach significance $(\gamma=$ $\left.0.02, t(10782)=0.75, p=.45, R_{m}^{2}<.001\right)$. Instead, we observed a tendency for a general decrease in Depressive symptomatology over time $\left(\gamma=-0.03, t(10782)=1.68, p=.09, R_{m}^{2}=.002\right.$; Effect of Group: $\left.\gamma=-5.07, t(82)=1.66, p=.10, R_{m}^{2}=.005\right)$.

For Resilience, we observed a general increase over time $(\gamma=0.06, t(10782)=3.59, p<$ $\left..001, R_{m}^{2}=.005\right)$. The main effect of Group did not reach significance $(\gamma=0.74, t(82)=0.20, p=$ $\left..84, R_{m}^{2}<.001\right)$. However, we obtained a significant Time $\times$ Group interaction in favor of the active control group $\left(\gamma=-0.05, t(10782)=2.07, p=.04, R_{m}^{2}=.002\right)$. In contrast to our hypothesis, followup simple slope analyses suggest that in the active control condition resilience significantly improved over time $(\gamma=0.06, S E=0.02, p<.001)$, whereas the trend for improvement in resilience did not significantly differ from zero in the cognitive control training condition $(\gamma=0.01, S E=$ $0.01, p=.39$; see supplemental Table 2 for descriptive information on experience sampling scores for the Baseline-, Training-, and Post-training phase).

\section{Network Models}

\section{Temporal Networks}

Figure 4 depicts the temporal network models of the baseline-, training-, and post-training experience sampling phase for the cognitive control training and active control condition 
separately. The obtained baseline-, training-, and post-training network models of the cognitive control training condition show a density of $1.73,2.21$, and 2.21 , respectively. The active control condition yielded density scores of $1.85,1.91$, and 1.89 respectively, suggesting that overall network density remained similar within the active control condition. In contrast, within the cognitive control training condition we observed an increase in network density from baseline to training, which remained stable from training to post-training. This suggests that, considering the unique associations between each of the constructs of interest and the autocorrelations, cognitive control training increased the overall level of connectivity within the model.

Within the cognitive control training condition, visual inspection of the network model(s) obtained at baseline (Figure 4a), during the training phase (Figure 4b), and following training (Figure 4c), suggests that changes in the network structure occurred throughout the experience sampling period. The corresponding Structural Hamming Distances for the comparison of the cognitive control training baseline network structure with the training- and post-training network structures were 7 and 2 respectively, suggesting that most of the changes in network structure in the cognitive control training condition reflect direct effects of training (i.e., differences in network structure that only occurred during the training phase, where following discontinuation of training, the network structure returned to its baseline state). However, this was not supported by statistical comparisons of network structure(s) on a single edge level. That is, after controlling for multiple comparisons, only one marginal significant edge difference was identified in the temporal network models, comparing the baseline network with the training- and post-training network. Compared to the baseline network, during cognitive control training a negative relation emerged between Rumination and Positive Appraisal ( $p=.002$; all other $p s \geq .028$ ), suggesting more subsequent use of Positive Appraisal at time $t+1$ when low levels of Rumination occurred at time $t$. In contrast, 
high levels of Rumination at time t predicted less subsequent use of Positive Appraisal. No significant edge differences were identified when comparing the baseline temporal network with the post-training temporal network (all $p s \geq .042$ ).

For the active control condition, the obtained network structure for the training phase (Figure 4e) showed relatively high similarity with the baseline structure (Figure 4d; Structural Hamming Distance $=3$ ). Instead, the post-training phase yielded a network structure that showed less similarity with the baseline network model (Figure 4f; Structural Hamming Distance $=8$ ). However, after correction for multiple comparisons, there were no significant edge differences between the baseline- and training network (all $p \mathrm{~s} \geq .049$ ) or baseline and post-training network (all $p \mathrm{~s} \geq .091)$.

Using Structural Hamming Distances, comparisons between both conditions in terms of their baseline-, training-, and post-training network structures suggested that most differences between groups occurred during the training phase (Structural Hamming Distance $=7$ ), whereas the Structural Hamming Distances for group differences in the baseline- and post-training network structures were 5 and 3 respectively. Noteworthy, after controlling for multiple comparisons, no statistically significant edge differences emerged between the cognitive control training and active control condition on a single edge level (all $p s \geq .019$ ).

\section{Contemporaneous Networks}

Effects of training condition on the contemporaneous network models are plotted in Figure 5. The observed density for the baseline- (Figure 5a), training- (Figure 5b), and post-training networks (Figure 5c) were 1.58, 1.68, and 1.62 for the cognitive control training condition. The obtained contemporaneous network models for the active control condition showed densities of 
$1.64,1.57$, and 1.59 respectively (Figures $5 \mathrm{~d}-\mathrm{f}$ ). This suggests that density scores remained relatively stable over time for the contemporaneous networks. In addition, both groups obtained highly similar density scores.

Moreover, the structure of the contemporaneous network models remained fairly stable over time. That is, for the cognitive control training condition, comparison of the baseline network structure with the training- and post-training network structures yielded Structural Hamming Distances of 3. For the active control condition, this yielded Structural Hamming Distances of 3 and 2 respectively. In line with these findings, within both training conditions, no significant edge differences emerged over time (all $p \mathrm{~s} \geq .38$ ).

Comparison of the network models between groups yielded Structural Hamming Distances of 3, 3, and 2 for the baseline-, training-, and post-training network structures respectively. Again, no significant edge differences were observed between both groups (all $p \mathrm{~s} \geq .56$ ).

\section{Discussion}

Previous studies suggest that cognitive control training holds potential as a preventive intervention for depression, where beneficial effects have been observed on maladaptive emotion regulation, residual symptomatology, and resilience (e.g., Hoorelbeke \& Koster, 2017). However, the mechanism underlying these emotional transfer effects remains mainly untested. That is, as to date no study has tested how experimental manipulation of cognitive control affects the complex interaction between affect, emotion regulation, and other indicators of functioning. Such knowledge is crucial to optimize deployment of cognitive control training as a strategy to reduce depression vulnerability, while also being informative for theoretical frameworks of cognitive risk for depression. To address this gap in the literature, the current study set out to model direct effects 
of cognitive control training on daily life emotion regulation dynamics, residual symptomatology, and resilience in formerly depressed patients, as this may increase our understanding of etiological pathways towards rumination as a depressive risk factor - rather than use of more adaptive emotion regulation strategies, which have been linked to resilience -, and how this can be treated effectively. For this purpose, we combined a four-week experience sampling period with a well-established experimental manipulation of cognitive control (Siegle et al., 2007; for a review, see Koster et al., 2017), randomizing patients with a history of depression over an adaptive PASAT (cognitive control) or speed-of-response (active control) training condition.

As a first step, building on earlier findings suggesting effects of cognitive control training on emotion regulation dynamics in a convenience sample (Hoorelbeke, Koster, et al., 2016), we modeled immediate effects of cognitive control training on deployment and efficacy of rumination and positive appraisal in daily life. In line with our expectations, cognitive control training impacted deployment of rumination. Our results suggest that, compared to the active control condition, the cognitive control training condition showed a stronger decrease in deployment of rumination over time. Noteworthy, this was contingent on affective state: the decrease in deployment of rumination for the cognitive control training condition over time became apparent in situations where participants were in a relatively positive mood state (i.e., at mean levels of affect and +2 SDs above one's individual mean), whereas deployment of rumination remained relatively stable over time in situations in which one's affective state was situated around 2 SDs below the individual mean.

These findings seem to suggest that immediate protective effects of cognitive control training on emotion regulation may show a mood congruent effect, where participants may initially not be able to override existing patterns of differential activation reflecting increased cognitive risk 
for depression (cf. Teasdale, 1988). As such, it does not seem to be the case that cognitive control training allows to fully override habitual ruminative tendencies upon confrontation with stress. Instead, our findings suggest that interventions such as cognitive control training, aimed at remediating cognitive control deficits, allow participants to exert more control over thought processes in absence of stressors, allowing to do better when already feeling relatively good. This is partially inconsistent with the notion that the working mechanism underlying effects of cognitive control training is that participants are able to engage less in rumination upon confrontation with stress.

It is important to note that the current study has focused on modeling immediate effects of cognitive control training on emotion regulation dynamics, where our pattern of findings may suggest a transition from inflexible habitual use of rumination in a context-independent manneralso referred to as ruminative inertia, which has recently been linked to reduced autonomic flexibility (Stange et al., 2020), use of other maladaptive emotion regulation strategies (Bean et al., in press) and elevated depressive symptomatology (Bean et al., 2020, in press) - towards more flexible use of this maladaptive emotion regulation strategy. As such, the possibility exists that the initial training gains on deployment of rumination may cascade into more robust changes following extended practice with emotion regulation and, related to this, mastery experience in not engaging in habitual rumination (e.g., in line with newly acquired relaxation techniques which are typically first practiced repeatedly in non-stressful situations before one is able to apply them in the context of stress). Indeed, several studies suggest that beneficial effects of cognitive control training seem to develop in the weeks and months following training (e.g., Hoorelbeke \& Koster, 2017; Vervaeke et al., 2021). Related to this, the possibility exists that in order to be able to effectively cope with stressors, more extensive training would be necessary. That is, although we have relied on a 
frequently used training scheme which has repeatedly shown to result in emotional transfer (e.g., Hoorelbeke \& Koster, 2017; Hoorelbeke, Koster, et al., 2015, 2016), as to date the relation between training intensity and emotional transfer following cognitive control training remains to be tested. As such, it is possible that more profound results could be acquired using a more intensive training strategy. However, identification of ideal training dosage would require experimental manipulation of amount of training sessions, using a wide range of training intensity. Here, individuals are likely to show strong heterogeneity in training needs. The observed lack of transfer of cognitive control training to deployment of rumination in more negative affective states, may also be related to the type of stimuli used in the current study, where cognitive control training procedures containing emotional stimuli may potentially yield broader transfer effects (e.g., Iacoviello et al., 2014, 2018; Schweizer et al., 2011).

Related to this, in line with prior findings, completing 10 sessions of the adaptive PASAT training did not impact deployment or efficacy of positive appraisal (Hoorelbeke, Koster, et al., 2016). Indeed, except for one study demonstrating beneficial effects of adaptive PASAT training on indicators of adaptive emotion regulation (Peckham \& Johnson, 2018), and previous findings suggesting beneficial effects of cognitive control training on resilience (Hoorelbeke \& Koster, 2017), transfer effects of cognitive control training have typically been limited to indicators of depression vulnerability (e.g., rumination, depressive symptomatology). Multiple factors may have contributed to this, where it has been suggested that difficulties with adaptive emotion regulation strategies are mostly apparent in severely disturbed clinical samples (Dillon \& Pizzagalli, 2013), whereas our sample consisted of remitted depressed patients. In addition, recent work has shown that clinical status is related to differences in how emotion regulation strategies interact to predict well-being. For instance, in a sample suffering from generalized anxiety disorder, Plate and 
colleagues (2016) observed a negative association between reappraisal and disability given low levels of non-acceptance, whereas in a healthy control group reappraisal was related to lower disability given high levels of non-acceptance. As such, adaptive emotion regulation strategies may compensate for negative effects of maladaptive emotion regulation in healthy samples. Instead, it has been argued that clinical samples may experience limited benefits from the use of adaptive emotion regulation strategies due to the presence of maladaptive emotion regulation strategies (Aldao \& Nolen-Hoeksema, 2012; Plate et al., 2016). This points towards the importance of considering how different emotion regulation strategies interact. Except for the network analyses exploring the unique associations between each of the constructs, in line with the pre-registration, we investigated effects of cognitive control training on emotion regulation dynamics within each strategy separately. Related to this, it should also be noted that, although we approached positive appraisal as an adaptive strategy, the adaptive nature of this process is contingent on the flexibility with which the emotion regulation strategy can be used to reach one's goals, and the appropriateness given a specific context (Aldao et al., 2015). Cognitive control deficits are likely to result in reduced emotion regulation flexibility and goal achievement. In line with this, recent findings suggest the presence of contextual attention inflexibility in subclinical depression (Godara et al., 2020), pointing towards the potential of incorporating procedures targeting attention flexibility (Godara et al., 2021).

It is possible that - in order to obtain more profound effects on emotion regulation cognitive control training would need to be combined with alternative interventions targeting emotion regulation. That is, while our findings suggest that remediating cognitive control may reduce the likelihood of deploying rumination, this does not imply that the subject has acquired necessary skills or insights to flexibly adopt alternative emotion regulation strategies. Here, factors 
such as emotional intelligence and meta-cognitions regarding emotion regulation strategies may also play an important role (e.g., Peña-Sarrionandia et al., 2015), as well as ability and prior experience with emotion regulation strategies. For this purpose, combining cognitive control training with emotion regulation training would be interesting.

The latter approach may also appeal to researchers and clinicians for a second reason, as our findings seem to suggest that there may be a potential downside to increased cognitive control. That is, the experimental manipulation significantly affected efficacy of maladaptive emotion regulation, suggesting an increased impact of rumination on affect over time in the cognitive control training condition relative to the active control group. Indeed, in contrast to the control condition, the relation between rumination and affect tended to become stronger over time for the cognitive control training group, where high levels of rumination predicted a more profound disruption of one's future affective state. These results significantly extend findings from prior prospective and experimental studies (e.g., Hoorelbeke, Koster, et al., 2016; Pe, Raes, et al., 2013) and are in line with the notion that increasing cognitive control may have detrimental effects when these regained cognitive resources are used to more strongly engage in maladaptive emotion regulation strategies and/or other dysfunctional behavior (e.g., based on positive metacognitions regarding rumination). Indeed, it has been argued that there is merit in combining cognitive control training procedures with interventions directly targeting maladaptive emotion regulation processes (Van den Bergh et al., 2018). For instance, a recent study focusing on the combination of cognitive control training and mindfulness suggested superior effects of a combined approach for worrying (Course-Choi et al., 2017).

The observation that cognitive control training impacted efficacy of maladaptive emotion regulation may also account for the unexpected pattern of results that was obtained for our second 
research question. That is, as a second step, we modeled immediate effects of cognitive control training (i.e., during the experience sampling period including the experimental manipulation of cognitive control) on self-reported residual cognitive- and depressive symptomatology, as well as resilience in daily life. In contrast to our expectations, no immediate beneficial effects of cognitive control training were observed for any of these outcome measures. Instead, we observed a (tendency for) a general decrease in cognitive complaints and depressive symptomatology during the experience sampling period, which did not significantly differ between both conditions. This may suggest limited immediate effects of cognitive control training on residual symptomatology and daily functioning. At the same time, however, these null-findings are in line with previous studies suggesting that effects of cognitive control training on cognitive complaints and depressive symptomatology may develop with a delayed onset, following immediate effects on emotion regulation processes (e.g., Hoorelbeke \& Koster, 2017; Siegle et al., 2014). In line with this, the long-term transfer effects of the current study suggest that adaptive PASAT training significantly lowered the risk for recurrence of depression over a period of one year follow-up (Hoorelbeke et al., 2021). Noteworthy, we observed a significant increase in resilience over time in the active control condition, relative to the cognitive control training condition. These immediate emotional transfer effects seem to be at odds with prior studies suggesting increased resilience three months following cognitive control training (Hoorelbeke \& Koster, 2017). A potential explanation for this observed discrepancy is the way in which resilience was conceptualized and assessed in the current study. That is, the resilience item prompts participants to indicate to what extent they felt able to successfully cope with stressors in daily life, which has shown to be strongly determined by affective state in remitted depressed patients (Hoorelbeke et al., 2019). Indeed, positive affect has been recognized as one of the main resilience factors. Related to this, the observed increase in emotion regulation efficacy during cognitive control training implies that participants who relied 
on rumination as a habitual style of repetitive negative thinking, were less likely to experience 'successful' coping with stressors in daily life. That is, the cost of ruminating in terms of impact on affect became more profound, while cognitive control training initially only affected deployment of rumination when participants were already in a relatively positive affective state. Together, these immediate effects may have resulted in relatively lower self-reported resilience in the cognitive control training condition compared to the active control condition.

We did not find differential effects of training condition on cognitive complaints and depressive symptomatology. Instead, both groups improved over time, which may be due to common factors of the assessment and training procedure. For instance, based on observed changes in variability in responses to items from experience sampling procedures over time, it has been emphasized that such procedures require an extensive familiarization period (Vachon et al., 2016). In addition, the operationalization of the comparison condition may also have contributed to this. That is, in both conditions broader cognitive functions (e.g., attentional processes) were trained, which may also impact residual symptomatology.

In order to gain further insight into the mechanisms underlying effects of cognitive control training, we proceeded with network analyses, modeling the unique interactions between emotion regulation, cognitive complaints, depressive symptomatology, and resilience over time. On a descriptive level, most deviations from the baseline structure of the temporal network occurred during the training phase in the cognitive control training condition (cf. Structural Hamming Distance values). However, statistical comparisons between both groups on a single edge level yielded no significant group differences after controlling for multiple comparisons. Interestingly, within the cognitive control training condition a significant inhibitory pathway between rumination and positive appraisal appeared, suggesting reduced future deployment of positive appraisal when 
being in a high ruminative state. This is in line with the observed effect of cognitive control training on efficacy of rumination. That is, deployment of positive appraisal has shown to be contingent on affect, which may be affected by the increased efficacy of rumination throughout the experimental manipulation. This may also potentially explain the unexpected findings regarding level of network density. That is, on a descriptive level, we noticed an increase in network density during the cognitive control training procedure. In this context, it has previously been suggested that higher levels of connectivity within a symptom network may reflect increased depression vulnerability (Cramer et al., 2016; van Borkulo et al., 2015). At the same time, however, recent findings suggest that this may not necessarily be the case. For instance, in a recent study in which cross-sectional depression networks obtained from patients suffering from major depressive disorder were compared to healthy controls, no differences in network density were observed (Hakulinen et al., 2020). Moreover, consistent with our descriptive findings suggesting an increase in network density during the intervention period, Bos and colleagues (2018) and Berlim and colleagues (2020) observed an increase rather than decrease in density of cross-sectional symptom networks following antidepressant treatment. In addition, previous studies exploring (pre - post) effects of interventions on dynamic network models consisting of mental states (e.g., momentary affect and worry) have yielded mixed results. For instance, comparing the impact of Mindfulness-Based Cognitive Therapy and antidepressant medication (with a waiting list- or placebo condition respectively), Snippe et al. (2017) observed no change in level of network connectivity following antidepressant treatment, whereas a decrease in network connectivity was observed following Mindfulness Based Cognitive Therapy. However, the same pattern of findings was observed in the waiting list control condition. 
Noteworthy, the contemporaneous network models showed high similarity over time and no within- or between group differences emerged on a single edge level, suggesting that patterns of co-occurring activity for the different cognitive risk- and protective factors included in the models were not modified by the experimental manipulation. In this context, it is interesting to note that other studies have also reported limited effects of other, well-established interventions for depression on dynamic network structures (e.g., Snippe et al., 2017).

This study is the first to model direct effects of cognitive control training on the complex interplay between emotion regulation dynamics, residual symptomatology, and resilience in remitted depressed patients in daily life. Moreover, although recent studies have explored whether routine care interventions can affect network structures (e.g., comparing pre- vs. post network models), as to our knowledge this study is the first to apply network analysis to model direct effects of an experimental manipulation as a means to map underlying mechanisms of change. This has yielded interesting findings, suggesting that direct effects of cognitive control training may be limited to impacting emotion regulation dynamics, and rumination in particular, where this does not result in immediate beneficial effects on depressive symptomatology, cognitive complaints, or resilience. Furthermore, the observation that cognitive control forms a protective factor, preventing deployment of rumination mostly when being in a relative positive affective state, has important consequences to how we measure effects of cognitive interventions on emotion regulation. That is, routinely used emotion regulation questionnaires typically instruct participants to indicate to what extent they use a given emotion regulation strategy, such as rumination, when being confronted with stressful situations or a negative affective state. However, our results suggest no immediate impact given those circumstances. This may have contributed to existing inconsistencies in the cognitive control training literature and warrants further investigation. Related to this, previous 
studies suggest that beneficial effects of adaptive PASAT training on depressive symptoms in remitted depressed patients are only partially mediated by change in depressive rumination (Hoorelbeke \& Koster, 2017). Hence, it is likely that other factors than rumination may also contribute to previously observed beneficial effects of preventive cognitive control training in the context of depression vulnerability. For instance, recent findings indicate that distress tolerance, the ability to persist in goal-related activities despite distress, may be involved in the mechanism underlying effects of cognitive control training (Lass et al., 2021). As to date, this transdiagnostic risk factor for psychopathology has received relatively little attention in the context of cognitive risk for depression (Lass \& Winer, 2020). However, in a sample of 72 students showing elevated depressive symptoms which were randomized to a waitlist condition or modifications of the adaptive PASAT, change in distress tolerance has recently been linked to both change in rumination and depressive symptomatology over time (Lass et al., 2021).

Although the current study offers an important methodological contribution to the cognitive training literature as a proof of principle study with a strong mechanistic focus, at the same time several limitations should be taken into account for future research. First, the use of experience sampling allows to model effects of cognitive control training on emotion regulation dynamics in a highly ecologically valid manner. At the same time, however, prolonged experience sampling procedures come at a cost of being highly intensive. As such, in the current study we kept the number of items at a minimum, relying on one-item solutions which may not fully represent the complexity of each of the constructs of interest. Moreover, although we made considerable efforts to familiarize participants with these items and validated the items at one year follow-up (Hoorelbeke et al., 2021), we cannot fully rule out individual differences in the interpretation of the items, which may also have been subject to change over time. Furthermore, in order to foster 
compliance to the experience sampling procedure, the baseline- and post-training assessment period were each limited to a seven day experience sampling period. In addition, ideally we would also have included multiple waves of follow-up experience sampling procedures using a measurement burst design, as this would allow to test whether effects of cognitive control training on emotion regulation dynamics would be maintained at later stages (e.g., three months followup), as well as model the influence of these parameters on other indicators of functioning over time. Related to this, although effects of cognitive control training on other indicators of emotion regulation could have been explored, in line with our pre-registration, we limited the analyses of emotion regulation dynamics to deployment and efficacy of rumination and positive appraisal based on prior research (e.g., Hoorelbeke, Koster, et al., 2016). Future studies may explore effects of cognitive control training on a broader range of emotion regulation strategies, as well as may consider to extend analyses of effects of cognitive control training on other indicators of functioning (e.g., inertia, flexibility; Aldao et al., 2015; Kuppens et al., 2010). Moreover, in case of inclusion of appropriate items in future studies, the methodology presented in the current manuscript would allow to test for alternative mechanisms underlying direct effects of adaptive PASAT training (e.g., distress tolerance; Lass et al., 2021) or other interventions. Third, to examine the causal influence of cognitive control on key depressive risk- and protective factors following remission of depression, we used a nomothetic approach in line with our pre-registration. This resulted in baseline-, training-, and post-training network models for each of the intervention conditions. The analytical method used in the current study does not allow to statistically test for significant differences in overall network structure (e.g., as implemented in the Network Comparison Test for network models obtained from cross-sectional data; van Borkulo et al., 2017). Instead, we provided a descriptive evaluation of the impact of the intervention on overall network structure (relying on the Structural Hamming Distance) and network density, whereas evaluation 
of statistical differences between both training conditions and within a given training condition over time took place on a single edge level. This resulted in loss of power to detect changes in the network structures due to correction for multiple comparisons. In addition, the current study strictly relied on self-report measures to model immediate effects of our experimental manipulation of cognitive control. In this context, it would be interesting for future studies to combine self-report measures with indicators of functioning assessed at different levels (e.g., combining self-report and behavioral measures within a network; Hoorelbeke, Marchetti, et al., 2016; Bernstein et al., 2017), which may potentially further advance our understanding of the relation between cognitive control and emotion regulation.

\section{Conclusion}

The current study set out to investigate the mechanisms underlying previously reported beneficial effects of cognitive control training as a preventive intervention for depression. For this purpose, we modeled effects of a widely used cognitive control training procedure on daily life emotion regulation dynamics, and explored effects of cognitive control training on the complex interplay between residual symptomatology, emotion regulation, and resilience in formerly depressed patients. Overall, effects on functioning in daily life were limited. Compared to an active control condition, cognitive control training demonstrated beneficial effects on deployment of rumination, albeit that direct effects of cognitive control training were restricted to limiting use of rumination when being in a fairly positive state. Interestingly, we observed a tendency for cognitive control training to impact efficacy of rumination, suggesting that there may be benefit in combining cognitive control training with other emotion regulation training procedures. Related to this, in contrast to our expectations, no immediate beneficial effects of cognitive control training were observed on other indicators of daily functioning. Furthermore, cognitive control training did not 
significantly impact the dynamic interplay between emotion regulation, residual symptomatology, and resilience.

\section{Declarations of Interest}

None.

\section{Acknowledgements}

We would like to thank Bo Swinnen, Lazlo Van Praet, Maarten Annot, and Yana Hertegonne for practical assistance. We would like to thank dr. Laura Bringmann for her helpful advice regarding analysis of the time series data.

\section{Funding}

This research was supported by an Applied Biomedical (TBM) grant of the Agency for Innovation through Science and Technology (IWT) of the Research Foundation-Flanders (FWO), awarded to the PrevenD project (IWT/TBM/150200). KH is a Postdoctoral Fellow of the FWO (FWO.3EO.2018.0031.01). EHW, RDR, and NVDB are funded by a Concerted Research Action Grant of Ghent University (Grant BOF16/GOA/017).

\section{References}

Acid, S., \& de Campos, L.M. (2003). Searching for bayesian network structures in the space of restricted acyclic partially directed graphs. Journal of Artificial Intelligence Research, 18, $445-490$.

Aldao, A., \& Nolen-Hoeksema, S. (2012). When are adaptive strategies most predictive of psychopathology? Journal of Abnormal Psychology, 121, 276-281. doi:10.1037/a0023598. 
Aldao, A., Sheppes, G., \& Gross, J.J. (2015). Emotion regulation flexibility. Cognitive Therapy and Research, 39, 263-278.

Bean, C.A.L., Heggeness, L.F., \& Ciesla, J.A. (in press). Ruminative inertia, emotion regulation, and depression: A daily-diary study. Behavior Therapy. doi: 10.1016/j.beth.2021.04.004

Bean, C.A.L., Heggeness, L.F., Kalmbach, D.A., \& Ciesla, J.A. (2020). Ruminative inertia and its association with current severity and lifetime course of depression. Clinical Psychological Science, 8, 1007-1016. doi: 10.1177/2167702620949174

Beloe, P., \& Derakhshan, N. (2019). Adaptive working memory training can reduce anxiety and depression vulnerability in adolescents. Developmental Science, e12831.

Berlim, M.T., Richard-Devantoy, S., Rodrigues Dos Santos, N., \& Turecki, G. (2020). The network structure of core depressive symptom domains in major depressive disorder following antidepressant treatment: A randomized clinical trial. doi: 10.1017/S0033291720001002

Bernstein, E.E., Heeren, A., \& McNally, R.J. (2017). Unpacking rumination and executive control: A network perspective. Clinical Psychological Science, 5, 816-826.

Borsboom, D., \& Cramer, A.O. (2013) Network analysis: An integrative approach to the structure of psychopathology. Annu Rev Clin Psychol., 9, 91-121.

Bos, F.M., Fried, E.I., Hollon, S.D., Bringmann, L.F., Dimidjian, S., DeRubeis, R.J., \& Bockting, C.L.H. (2018). Cross-sectional networks of depressive symptoms before and after antidepressant medication treatment. Social Psychiatry and Psychiatric Epidemiology, 53, 617-627. doi: 10.1007/s00127-018-1506-1

Braver, T.S. , Cohen, J.D., \& Barch, D.M. (2002). The Role of the Prefrontal Cortex in Normal and Disordered Cognitive Control: a Cognitive Neuroscience Perspective. Oxford, UK: Oxford University Press. 
Bringmann, L.F., Pe, M.L., Vissers, N., Ceulemans, E., Borsboom, D., Vanpaemel, W., Tuerlinckx, F., \& Kuppens, P. (2016). Assessing temporal emotion dynamics using networks. Assessment, 23, 425-435.

Brunoni, A., Boggio, P., De Raedt, R., Benseñor, I., Lotufo, P., Namur, V., Valiengo, L.C.L., \& Vanderhasselt, M.-A. (2014). Cognitive control therapy and transcranial direct current stimulation for depression: a randomized, double-blinded, controlled trial. Journal of Affective Disorders, 162, 43-49.

Calkins, A. W., McMorran, K. E., Siegle, G. J., \& Otto, M. W. (2015). The effects of computerized cognitive control training on community adults with depressed mood. Behavioural and cognitive psychotherapy, 43, 578-589.

Cohen, J. (2017). Cognitive control: core constructs and current considerations. The Wiley Handbook of Cognitive Control Chichester, West Sussex, UK: John Wiley and Sons.

Cohen, N., Margulies, D.S., Ashkenazi, S., Schaefer, A., Taubert, M., Henik, A., Villringer, A., \& Okon-Singer, H. (2016). Using executive control training to suppress amygdala reactivity to aversive information. NeuroImage, 125, 1022-1031.

Course-Choi, J., Saville, H., \& Derakshan, N. (2017). The effects of adaptive working memory training and mindfulness meditation training on processing efficiency and worry in high worriers. Behavior Research and Therapy, 89, 1-13.

Cramer, A. O. J., van Borkulo, C. D., Giltay, E. J., van der Maas, H. L. J., Kendler, K. S., Scheffer, M., \& Borsboom, D. (2016). Major depression as a complex dynamic system. PLoS ONE, 11, e0167490, doi: 10.1371/journal.pone.0167490

Demeyer, I., De Lissnyder, E., Koster, E.H.W., \& De Raedt, R. (2012). Rumination mediates the relationship between impaired cognitive control for emotional information and depressive 
symptoms: A prospective study in remitted depressed adults. Behaviour Research and Therapy, 50, 292-297. doi: 10.1016/j.brat.2012.02.012

De Raedt, R., \& Koster, E.H.W. (2010). Understanding vulnerability for depression from a cognitive neuroscience perspective: A reappraisal of attentional factors and a new conceptual framework. Cognitive Affective \& Behavioral Neuroscience, 10, 50-70.

Devilly, G.J., \& Borkovec, T.D. (2000). Psychometric properties of the credibility/expectancy questionnaire. Journal of behavior therapy and experimental psychiatry, 31(2), 73-86.

Dillon, D.G., \& Pizzagalli, D.A. (2013). Evidence of successful modulation of brain activation and subjective experience during reappraisal of negative emotion in unmedicated depression. Psychiatry Research, 212, 99-107.

Engelen, U., De Peuter, S., Victoir, A., Van Diest, I., \& Van den Bergh, O. (2006). Verdere validering van de Positive and Negative Affect Schedule (PANAS) en vergelijking van twee Nederlandstalige versies. Gedrag en gezondheid, 34, 61-70.

Epskamp, S., Cramer, A., Waldorp, L., Schmittmann, V. D., \& Borsboom, D. (2012). Qgraph: Network visualizations of relationships in psychometric data. Journal of Statistical Software, 48, 1-18.

Epskamp, S., Waldorp, L. J., Mõttus, R., \& Borsboom, D. (2018). The Gaussian Graphical Model in Cross-sectional and Time-series Data. Multivariate Behavioral Research, 1-28.

Garnefski, N., Kraaij, V., \& Spinhoven, P. (2001). Negative life events, cognitive emotion regulation and emotional problems. Personality and Individual Differences, 30, 1311-1327.

Godara, M., Sanchez Lopez, A., Baeken, C., \& De Raedt, R. (2021). Looking for carrots, watching out for sticks: a gaze-contingent approach towards training contextual goal-dependent affective attention flexibility. Behaviour Research and Therapy, 136. doi: 10.1016/j.brat.2020.103787 
Godara, M., Sanchez-Lopez, A., Liefooghe, B., \& De Raedt, R. (2020). Contextual changes influence attention flexibility towards new goals. Cognitive Therapy \& Research, 44, 327344. doi: $10.1007 / \mathrm{s} 10608-019-10072-5$

Gronwall, D. (1977). Paced auditory serial-addition task: a measure of recovery from concussion. Perceptual and motor skills, 44, 367-373.

Hakulinen, C., Fried, E.I., Pulkki-Råback, L., Virtanen, M., Suvisaari, J., \& Elovainio, M. (2020). Network structure of depression symptomology in participants with and without depressive disorder: the population-based Health 2000-2011 study. Social Psychiatry and Psychiatric Epidemiology, 55, 1273-1282. doi: 10.1007/s00127-020-01843-7

Harvey, P. O., Le Bastard, G., Pochon, J. B., Levy, R., Allilaire, J. F., Dubois, B., \& Fossti, P. (2004). Executive functions and updating of the contents of working memory in unipolar depression. Journal of Psychiatric Research, 38, 567-576. doi: 10.1016/j.jpsychires.2004.03.003

Hofmann, S.G., Curtis, J.C., \& McNally, R.J. (2016). A complex network perspective on clinical science. Perspect Psychol Sci., 11, 597-605.

Hofmann, W., \& Patel, P. V. (2015). SurveySignal: A convenient solution for experience sampling research using participants' own smartphones. Social Science Computer Review, 33, 235253.

Hoorelbeke, K., Faelens, L., Behiels, J., \& Koster, E.H.W. (2015). Internet-delivered cognitive control training as a preventive intervention for remitted depressed patients: protocol for a randomized controlled trial. Bmc Psychiatry, 15, 125.

Hoorelbeke, K., \& Koster, E.H.W. (2017). Internet-delivered cognitive control training as a preventive intervention for remitted depressed patients: Evidence from a double-blind 
randomized controlled trial study. Journal of consulting and clinical psychology, 85, 135146.

Hoorelbeke, K., Koster, E.H.W., Demeyer, I., Loeys, T., \& Vanderhasselt, M.-A. (2016). Effects of cognitive control training on the dynamics of (mal)adaptive emotion regulation in daily life. Emotion, 16, 945-956.

Hoorelbeke, K., Koster, E.H.W., Vanderhasselt, M.-A., Callewaert, S., \& Demeyer, I. (2015). The influence of cognitive control training on stress reactivity and rumination in response to a lab stressor and naturalistic stress. Behaviour research and therapy, 69, 1-10.

Hoorelbeke, K., Marchetti, I., De Schryver, M., \& Koster, E.H.W. (2016). The interplay between cognitive risk and resilience factors in remitted depression: A network analysis. Journal of Affective Disorders, 195, 96-104.

Hoorelbeke, K., Van den Bergh, N., Wichers, M., \& Koster, E. H. W. (2019). Between vulnerability and resilience: A network analysis of fluctuations in cognitive risk and protective factors following remission from depression. Behaviour Research and Therapy, 116, 1-9.

Hoorelbeke, K., Van den Bergh, N., De Raedt, R., Wichers, M., \& Koster, E. H. W. (2021). Preventing recurrence of depression: Long-term effects of a randomized controlled trial on cognitive control training for remitted depressed patients. Clinical Psychological Science, 9, 615-633. doi: 10.1177/2167702620979775

Hsu, K.J., Beard, C., Rifkin, L., Dillon, G.D., Pizzagalli, D.A., \& Björgvinsson, T. (2015). Transdiagnostic mechanisms in depression and anxiety: The role of rumination and attentional control. J Affect Disord., 188, 22-27.

Iacoviello, B.M., Huryk, K., Alvarez, E., Collins, K., Murrough, J., Iosifescu, D., \& Charney, D. (2014). Cognitive-emotional training as an intervention for major depressive disorder. Depression \& Anxiety, 31, 699-706. 
Iacoviello, B.M., Murrough, J.W., Hoch, M.W., Huryk, K.M., Collins, K.A., Cutter, G.R., Iosifescu, D.V., \& Charney, D.S. (2018). A randomized, controlled pilot trial of the emotional faces memory task: A digital therapeutic for depression. Npj Digital Medicine, $1,21$.

Jaeggi, S.M., Buschkuehl, M., Jonides, J., \& Perrig, W.J. (2008). Improving fluid intelligence with training on working memory. Proceedings of the National Academy of Sciences of the United States of America, 105, 6829-6833.

Joormann, J., \& Vanderlind, M. W. (2014). Emotion regulation in depression: The role of biased cognition and reduced cognitive control. Clinical Psychological Science, 2, 402-421.

Kalisch, R., Müller, M.B., \& Tuescher, O. (2015). A conceptual framework for the neurobiological study of resilience. Behavioral and Brain Sciences, 38, 1-21. doi: 10.1017/S0140525X1400082X

Koster, E.H.W., De Lissnyder, E., Derakshan, N., \& De Raedt, R., (2011). Understanding depressive rumination from a cognitive science perspective: The impaired disengagement hypothesis. Clin Psychol Rev., 31, 138-145.

Koster, E.H.W., Hoorelbeke, K., Onraedt, T., Owens, M., \& Derakshan, N. (2017). Cognitive control interventions for depression: A systematic review of findings from training studies. Clinical Psychology Review, 53, 79-92.

Kuppens, P., Allen, N.B., \& Sheeber, L.B. (2010). Emotional intertia and psychological maladjustment. Psychol Sci, 21, 984-991.

Larson, R., \& Csikszentmihalyi, M. (1983). The experience sampling method. New Directions for Methodology of Social and Behavioral Science, 15, 41-56. 
Lass, A.N.S., Rokke, P.D., \& Winer, E.S. (2021). Evaluating cognitive control training on symptoms of depression over time: Three potential mechanisms. Journal of Affective Disorders Reports, 4, 100127.

Lass, A.N.S., \& Winer, E.S. (2020). Distress tolerance and symptoms of depression: A review and integration of literatures. Clinical Psychology Science and Practice, 27, e12336. doi: $10.1111 /$ cpsp. 12336

Launder, N.H., Minkov, R., Davey, C.G., Finke, C., Gavelin, H.M., \& Lampit, A. (2021). Computerized cognitive training in people with depression: A systematic review and metaanalysis of randomized clinical trials. Preprint. doi: 10.1101/2021.03.23.21254003

Levens, S. M., \& Gotlib, I. H. (2015). Updating emotional content in recovered depressed individuals: Evaluating deficits in emotion processing following a depressive episode. Journal of behavior therapy and experimental psychiatry, 48, 156-163.

Miyake, A., Friedman, N.P., Emerson, M.J., Witzki, A.H., Howerter, A., \& Wager, T.D. (2000). The unity and diversity of executive functions and their contributions to complex "frontal lobe" tasks: A latent variable analysis. Cognitive Psychology, 41, 49-100.

Moberly, N.J., \& Watkins, E.R. (2008). Ruminative self-focus and negative affect: an experience sampling study. Journal of Abnormal Psychology, 117, 314-323.

Mor, N., \& Daches, S. (2015). Ruminative thinking: Lessons learned from cognitive training. Clinical Psychological Science, 3, 574-592.

Moshier, S.J., Molokotos, E., Stein, A.T., \& Otto, M.W. (2015). Assessing the effects of depressed mood and cognitive control training on memory confidence and accuracy following repeated checking. International Journal of Cognitive Therapy, 8, 1-16. 
Moshier, S.J., \& Otto, M.W. (2017). Behavioral activation treatment for major depression: A randomized trial of the efficacy of augmentation with cognitive control training. $J$ Affect Disord., 210, 265-268.

Motter, J. N., Pimontel, M. A., Rindskopf, D., Devanand, D. P., Doraiswamy, P. M., \& Sneed, J. R. (2016). Computerized cognitive training and functional recovery in major depressive disorder: a meta-analysis. Journal of Affective Disorders, 189, 184-191.

Nakagawa, S., \& Schielzeth, H. (2013). A general and simple method for obtaining R2 from generalized linear mixed effects models. Methods in Ecology and Evolution, 4, 133-142.

Nolen-Hoeksema, S., Wisco, B. E., \& Lyubomirsky, S. (2008). Rethinking rumination. Perspectives on Psychological Science, 3, 400-424. doi: 10.1111/j.17456924.2008.00088.x

Pe, M.L., Raes, F., \& Kuppens, P. (2013). The cognitive building blocks of emotion regulation: Ability to update working memory moderates the efficacy of rumination and reappraisal on emotion. PLoS One, 8, e69071.

Pe, M.L., Raes, F., Koval, P., Brans, K., Verduyn, P., \& Kuppens, P. (2013). Interference resolution moderates the impact of rumination and reappraisal on affective experiences in daily life. Cognition and Emotion, 27, 492-501.

Peckham, A.D., \& Johnson, S.L.J. (2018). Cognitive control training for emotion-related impulsivity. Behaviour Research and Therapy, 105, 17-26.

Peeters, F., Nicolson, N., Wichers, M., \& Hacker, P. (2013). Remission of Depression Questionnaire-Dutch translation $(R D Q-N L)$.

Peña-Sarrionandia, A., Mikolajczak, M., \& Gross, J.J. (2015). Integrating emotion regulation and emotional intelligence traditions: A meta-analysis. Frontiers in Psychology, 6, 160. 
Plate, A.J., Aldao, A., Quintero, J.M., \& Mennin, D.S. (2016). Interactions between reappraisal and emotional nonacceptance in psychopathology: Examining disability and depression symptoms in generalized anxiety disorder. Cognitive Therapy \& Research, 40, 733-746. doi: 10.1007/s10608-016-9793-x

Portzky, M. (2008). RS-nl: Resilience Scale-Nederlandse versie. In: Amsterdam: Harcourt Test Publishers.

Pruessner, L., Barnow, S., Holt, D.V., Joormann, J., \& Schulze, K. (2020). A cognitive control framework for understanding emotion regulation flexibility. Emotion, 20, 21-29

Scholte, E., \& Noens, I. (2011). BRIEF-A. In Vragenlijst executieve functies voor volwassenen. Handleiding. Amsterdam: Hogreve Uitgevers.

Schweizer, S., Hampshire, A., \& Dalgleish, T. (2011). Extending brain-training to the affective domain: Increasing cognitive and affective executive control through emotional working memory training. Plos One, 6, 7.

Segrave, R.A., Arnold, S., Hoy, K., \& Fitzgerald, P.B. (2014). Concurrent cognitive control training augments the antidepressant efficacy of tDCS: A pilot study. Brain Stimulation, 7, $325-331$.

Siegle, G.J., Ghinassi, F., \& Thase, M.E. (2007). Neurobehavioral therapies in the 21st century: summary of an emerging field and an extended example of cognitive control training for depression. Cognitive Therapy and Research, 31, 235-262.

Siegle, G.J., Price, R.B., Jones, N.P., Ghinassi, F., Painter, T., \& Thase, M.E. (2014). You gotta work at it: Pupillary indices of task focus are prognostic for response to a neurocognitive intervention for rumination in depression. Clinical Psychological Science, 2, 455-471. 
Snippe, E., Viechtbauer, W., Geschwind, N., Klippel, A., de Jong, P., \& Wichers, M. (2017). The impact of treatments for depression on the dynamic network structure of mental states: Two randomized controlled trials. Scientific Reports, 7, 46523.

Snyder, H.R., \& Hankin, B.L. (2016). Spiraling out of control: Stress generation and subsequent rumination mediate the link between poorer cognitive control and internalizing psyhopathology. Clinical Psychological Science, 4, 1047-1064.

Stange, J.P., Hamilton, J.L., Shepard, R., Wu, J., Fresco, D.M., \& Alloy, L.B. (2020). Inflexible autonomic responses to sadness predict habitual and real-world rumination: A multi-level, multi-wave study. Biological Psychology, 153, 107886. doi: 10.1016/j.biopsycho.2020.107886

Teasdale, J.D. (1988). Cognitive vulnerability to persistent depression. Cognition and Emotion, 2, 247-274.

Treynor, W., Gonzalez, R., \& Nolen-Hoeksema, S. (2003). Rumination reconsidered: A psychometric analysis. Cognitive Therapy and Research, 27, 247-259.

Vachon, H., Bourboussom, M., Deschamps, T., Doron, J. Bulteau, S., Sauvaget, A., \& ThomasOllivier, V. (2016). Repeated self-evaluations may involve familiarization: An exploratory study related to ecological momentary assessment designs in patients with major depressive disorder. Psychiatry Research, 245, 99-104. doi: 10.1016/j.psychres.2016.08.034

van Borkulo, C., Boschloo, L., Borsboom, D., Penninx, B.W., Waldorp, L.J., \& Schoevers R.A. (2015). Association of Symptom Network Structure With the Course of Depression. JAMA Psychiatry, 72, 1219-1226. doi: 10.1001/jamapsychiatry.2015.2079

van Borkulo, C.D., Boschloo, L., Kossakowski, J.J., Tio, P., Schoevers, R.A., Borsboom, D., \& Waldorp, L. J. W. (2017). Comparing network structures on three aspects: A permutation test. doi: 10.13140/RG.2.2.29455.38569. 
Van den Bergh, N., Hoorelbeke, K., De Raedt, R., \& Koster, E. H. (2018). Remediation of depression-related cognitive impairment: cognitive control training as treatment augmentation. Expert review of neurotherapeutics, 18, 907-913.

Van den Bergh, N., Vermeersch, S., Hoorelbeke, K., Vervaeke, J., De Raedt, R., \& Koster, E.H.W. (2020). Cognitive control training as an augmentation strategy to CBT in the treatment of fear of failure in undergraduates. Cognitive Therapy and Research, 44, 1199-1212.

Van der Does, A. (2002). BDI-II-NL. Handleiding. De Nederlandse versie van de Beck depression inventory. In: Lisse: Harcourt Test Publishers.

Vanderhasselt, M.-A., \& De Raedt, R. (2009). Impairments in cognitive control persist during remission from depression and are related to the number of past episodes: An event related potentials study. Biological Psychology, 81, 169-176.

Van Vliet, I., \& De Beurs, E. (2007). The MINI-International Neuropsychiatric Interview. A brief structured diagnostic psychiatric interview for DSM-IV en ICD-10 psychiatric disorders. Tijdschrift voor psychiatrie, 49, 393-397.

Vervaeke, J., Hoorelbeke, K., Baeken, C., \& Koster, E.H.W. (2021). Online cognitive control training for remitted depressed individuals: A replication and extension study. Cognitive Therapy and Research. doi: 10.1007/s10608-021-10238-0

Vervaeke, J., Hoorelbeke, K., Baeken, C., Van Looy, J., \& Koster, E.H.W. (2020). Transfer and Motivation After Cognitive Control Training for Remitted Depression in Healthy Sample. Journal of Cognitive Enhancement, 4, 49-61.

Wagnild, G., \& Young, H. (1993). Development and psychometric Evaluation of the Resilience Scale. Journal of nursing measurement, 1(2), 165-178.

Wagnild, R. N., 2009. A review of the Resilience Scale. Journal of Nursing Measurement, 17, 105113. 
Watkins, E.R., \& Roberts, H. (2020). Reflecting on rumination: Consequences, causes, mechanisms and treatment of rumination. Behaviour Research and Therapy, 127, 103573.

Weightman, M.J., Knight, M.J., \& Baune, B.T. (2019). A Systematic Review of the Impact of Social Cognitive Deficits on Psychosocial Functioning in Major Depressive Disorder and Opportunities for Therapeutic Intervention. Psychiatry research, 274, 195-212.

Zimmerman, M., Martinez, J.H., Attiullah, N., Friedman, M., Toba, C., Boerescu, D.A., \& Ragheb, M. (2013). A new type of scale for determining remission from depression: the remission from depression questionnaire. Journal of Psychiatric Research, 47, 78-82. 
Table 1

Group characteristics

\begin{tabular}{|c|c|c|c|c|}
\hline \multirow[b]{3}{*}{ Variables } & \multicolumn{4}{|c|}{ Training condition } \\
\hline & \multirow{2}{*}{\multicolumn{2}{|c|}{$\frac{\text { Cognitive control }(n=47)}{\text { Ratio } / \%}$}} & \multicolumn{2}{|c|}{ Active control $(n=45)$} \\
\hline & & & Ratio & \\
\hline Gender (male : female) & & $19: 28$ & & $14: 31$ \\
\hline Number of previous episodes (ratio: $1: 2: 3: \geq 4$ ) & & $: 10: 18$ & & $: 11: 19$ \\
\hline$\%$ reporting depressive episodes within a bipolar disorder & & $6 \%$ & & $7 \%$ \\
\hline$\%$ reporting current use of antidepressant medication & & $51 \%$ & & $49 \%$ \\
\hline$\%$ reporting current use of benzodiazepines & & $13 \%$ & & $22 \%$ \\
\hline$\%$ reporting current use of lithium & & $6 \%$ & & $4 \%$ \\
\hline \multirow{2}{*}{$\begin{array}{l}\% \text { reporting current use of antipsychotic or neuroleptic } \\
\text { medication }\end{array}$} & & $2 \%$ & & $2 \%$ \\
\hline & $M$ & $S D$ & $M$ & $S D$ \\
\hline Age & 45.74 & 14.21 & 45.84 & 11.40 \\
\hline Mean self-reported episode length in months (SD) & 7.27 & 9.94 & 6.36 & 7.74 \\
\hline $\begin{array}{l}\text { Mean self-reported time since previous episode in months } \\
\text { (SD) }\end{array}$ & 31.80 & 44.08 & 29.29 & 40.96 \\
\hline Cognitive control (non-adaptive PASAT) & 0.55 & 0.17 & 0.56 & 0.17 \\
\hline Depressive symptoms (BDI-II-NL) & 11.28 & 8.58 & 13.07 & 9.66 \\
\hline Residual symptoms (RDQ) & 22.40 & 16.49 & 24.87 & 14.38 \\
\hline Positive Affect (PANAS) & 34.77 & 7.12 & 33.33 & 7.20 \\
\hline Negative Affect (PANAS) & 23.15 & 6.70 & 25.42 & 6.07 \\
\hline Rumination (RRS total score) & 49.96 & 12.57 & 53.98 & 12.83 \\
\hline Brooding (RRS Brooding subscale) & 11.36 & 3.21 & 12.73 & 3.53 \\
\hline Reflection (RRS Reflection subscale) & 10.66 & 3.42 & 11.31 & 3.43 \\
\hline Adaptive Emotion Regulation (CERQ) & 65.23 & 13.27 & 61.33 & 16.73 \\
\hline Maladaptive Emotion Regulation (CERQ) & 38.85 & 7.61 & 40.44 & 9.19 \\
\hline Resilience (RS) & 73.06 & 11.64 & 74.42 & 11.13 \\
\hline Cognitive complaints (BRIEF) & 123.70 & 20.22 & 131.33 & 20.25 \\
\hline Credibility (CEQ) & 0.02 & 2.47 & -0.02 & 2.27 \\
\hline Expectancy (CEQ) & 0.15 & 2.67 & -0.16 & 2.59 \\
\hline
\end{tabular}


Note: No group differences emerged at baseline (all $p \mathrm{~s}>.05$ ). 
Table 2

Effects of Training Condition on Emotion Regulation Dynamics

\begin{tabular}{|c|c|c|c|c|c|}
\hline Prediction Effect & $\gamma$ & $S E$ & $t$ & $p$ & $R_{m}^{2}$ \\
\hline \multicolumn{6}{|l|}{ Deployment of Emotion Regulation } \\
\hline \multicolumn{6}{|l|}{ Prediction of RUM ${ }_{t}$ by Affect ${ }_{t-1}$} \\
\hline Time & -0.015 & 0.019 & -0.76 & .44 & $<.001$ \\
\hline Affect $t-1$ & -0.176 & 0.050 & -3.54 & $<.001$ & .001 \\
\hline Group & -4.069 & 3.362 & -1.21 & .23 & .002 \\
\hline Time $\times$ Affect $t-1$ & $<0.001$ & $<0.001$ & 0.47 & .64 & $<.001$ \\
\hline Time $\times$ Group & -0.019 & 0.026 & -0.72 & .47 & $<.001$ \\
\hline Affect $t-1 \times$ Group & 0.051 & 0.068 & 0.75 & .46 & $<.001$ \\
\hline Time $\times$ Affect $_{\mathrm{t}-1} \times$ Group & -0.002 & $<0.001$ & -2.78 & $<.01$ & $<.001$ \\
\hline Prediction of APP ${ }_{t}$ by Affect $t-1$ & & & & & .017 \\
\hline Time & 0.019 & 0.015 & 1.28 & .20 & $<.001$ \\
\hline Affect $t-1$ & 0.177 & 0.060 & 2.94 & $<.01$ & .001 \\
\hline Group & 3.085 & 4.147 & 0.74 & .46 & .001 \\
\hline Time $\times$ Affect $t-1$ & 0.002 & $<0.001$ & 3.15 & $<.01$ & .001 \\
\hline Time $\times$ Group & -0.029 & 0.020 & -1.41 & .16 & $<.001$ \\
\hline Affect $t-1 \times$ Group & -0.012 & 0.083 & -0.14 & .89 & $<.001$ \\
\hline Time $\times$ Affect $_{\mathrm{t}-1} \times$ Group & $<0.001$ & $<0.001$ & 0.11 & .91 & $<.001$ \\
\hline \multicolumn{6}{|l|}{ Efficacy of Emotion Regulation } \\
\hline Prediction of Affect ${ }_{t}$ by RUM $_{t}$ & & & & & .167 \\
\hline Time & 0.017 & 0.006 & 2.74 & $<.01$ & .001 \\
\hline $\mathrm{RUM}_{\mathrm{t}}$ & -0.298 & 0.028 & -10.66 & $<.001$ & .016 \\
\hline Group & 0.965 & 2.366 & 0.41 & .68 & $<.001$ \\
\hline Affect $t-1$ & 0.346 & 0.008 & 42.63 & $<.001$ & .056 \\
\hline Time $\times$ RUM $_{t}$ & 0.001 & $<0.001$ & 3.06 & $<.01$ & .001 \\
\hline Time $\times$ Group & -0.020 & 0.008 & -2.42 & .02 & .001 \\
\hline RUM $_{t} \times$ Group & 0.066 & 0.038 & 1.71 & .09 & $<.001$ \\
\hline Time $\times$ RUM $_{t} \times$ Group & -0.002 & $<0.001$ & -3.47 & $<.001$ & .002 \\
\hline Prediction of Affect ${ }_{t}$ by APP $_{t}$ & & & & & .199 \\
\hline Time & 0.015 & 0.007 & 2.19 & .03 & .001 \\
\hline $\mathrm{APP}_{t}$ & 0.324 & 0.027 & 12.06 & $<.001$ & .021 \\
\hline Group & 0.170 & 2.262 & 0.08 & .94 & $<.001$ \\
\hline Affect $t-1$ & 0.289 & 0.008 & 38.38 & $<.001$ & .043 \\
\hline Time $\times \mathrm{APP}_{t}$ & $<0.001$ & $<0.001$ & -1.03 & .30 & $<.001$ \\
\hline Time $\times$ Group & -0.006 & 0.009 & -0.68 & .50 & $<.001$ \\
\hline $\operatorname{APP}_{t} \times$ Group & -0.008 & 0.036 & -0.22 & .83 & $<.001$ \\
\hline Time $\times \mathrm{APP}_{t} \times$ Group & $<0.001$ & $<0.001$ & 0.30 & .77 & $<.001$ \\
\hline
\end{tabular}

Note: $\mathrm{RUM}=$ Rumination. $\mathrm{APP}=\mathrm{Positive}$ Appraisal. 


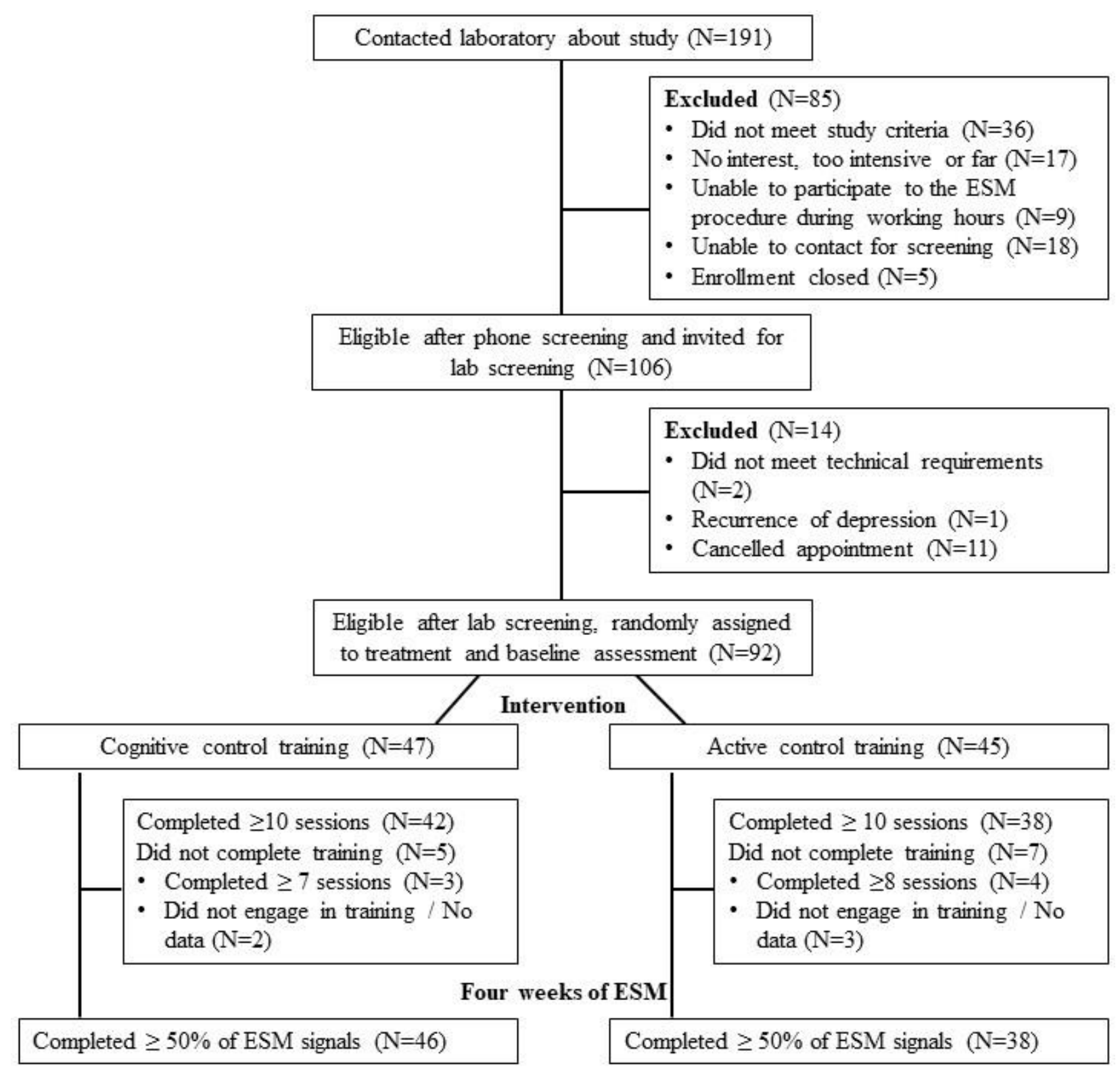

Figure 1. CONSORT Flow diagram

Note: $E S M=$ Experience Sampling Methodology 

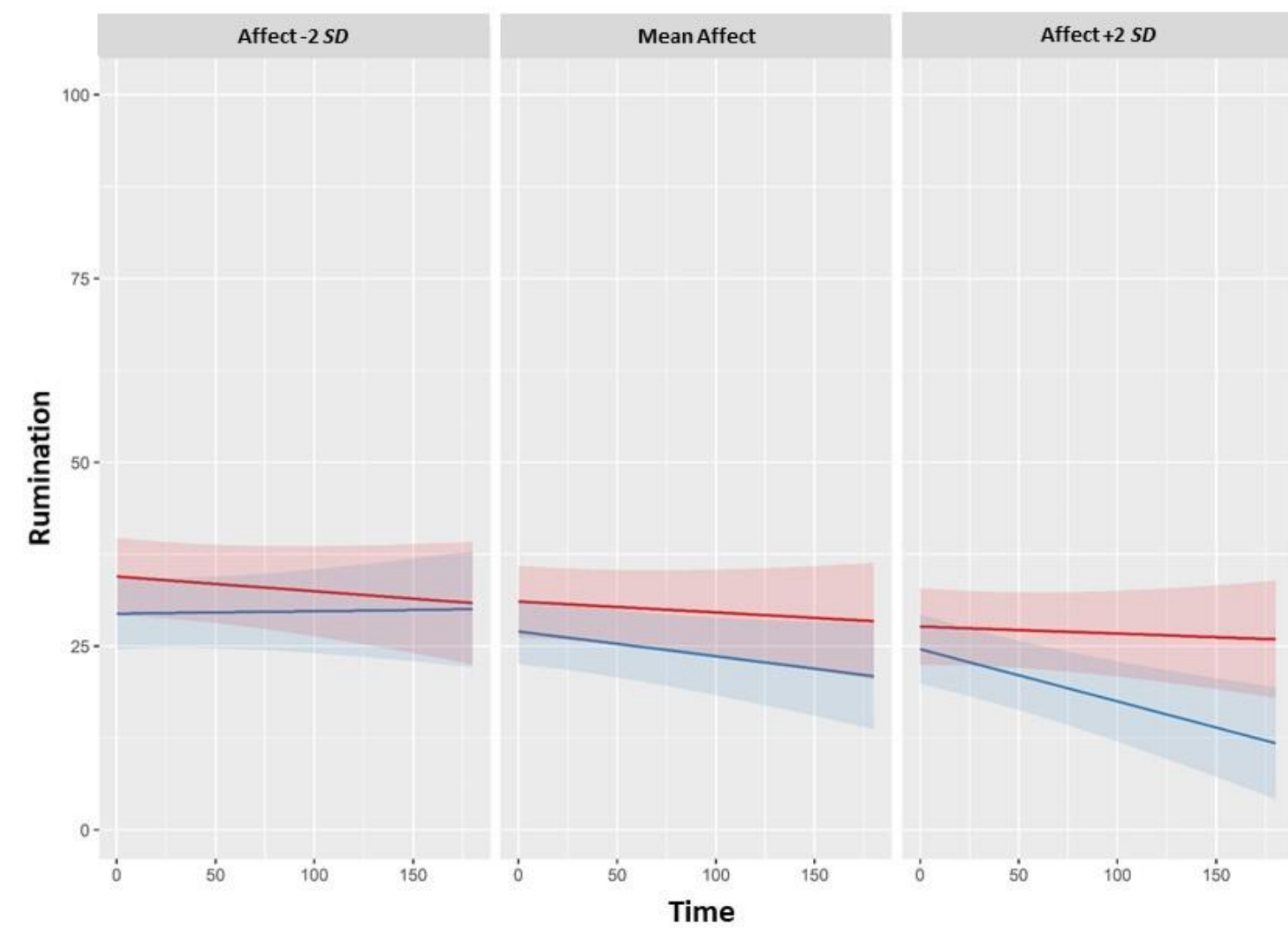

\section{Group}

Active control Cognitive control

Figure 2. Effects of cognitive control training on Deployment of Rumination 

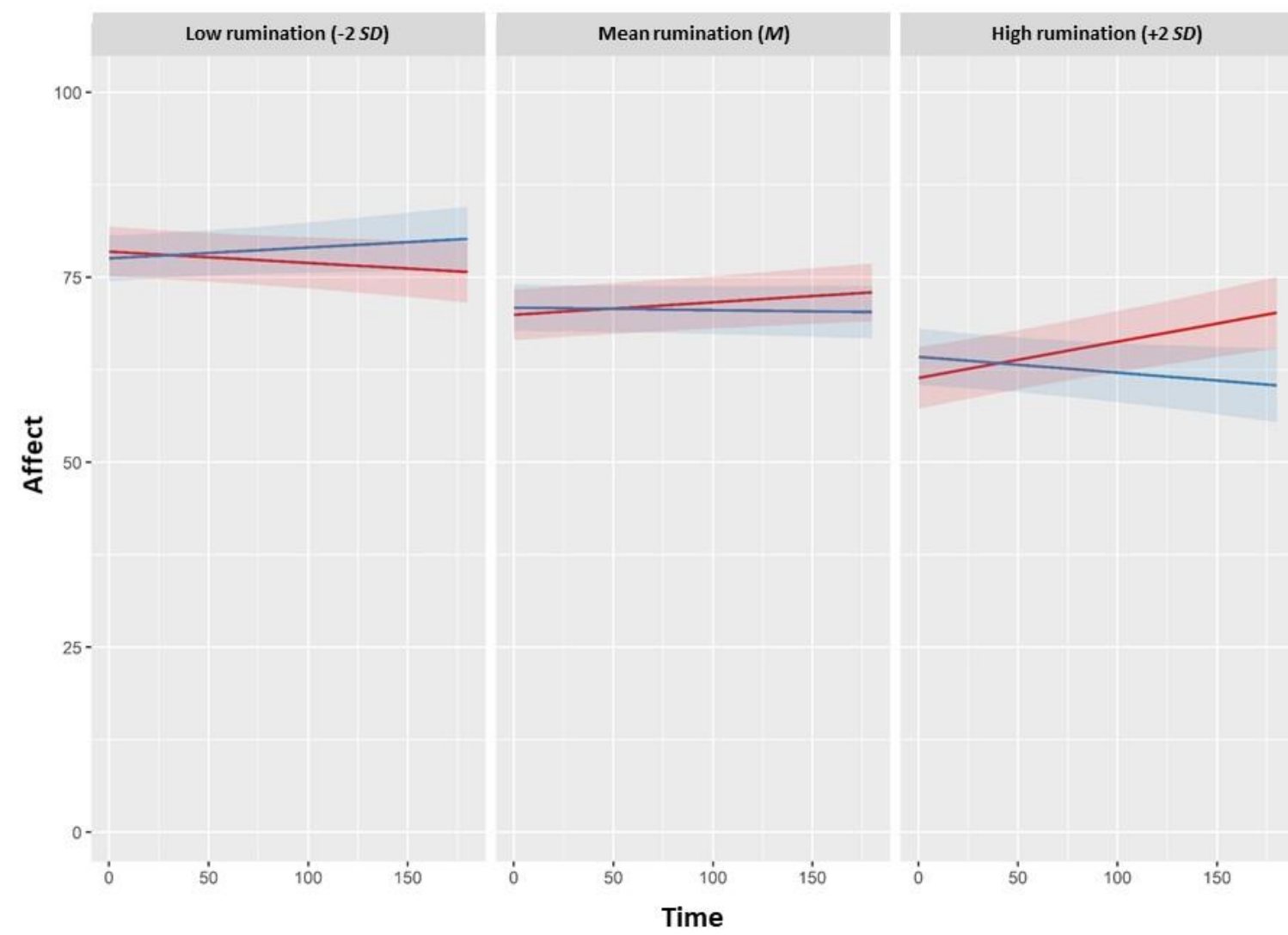

Group

- Active control Cognitive control

Figure 3. Effects of cognitive control training on Efficacy of Rumination 

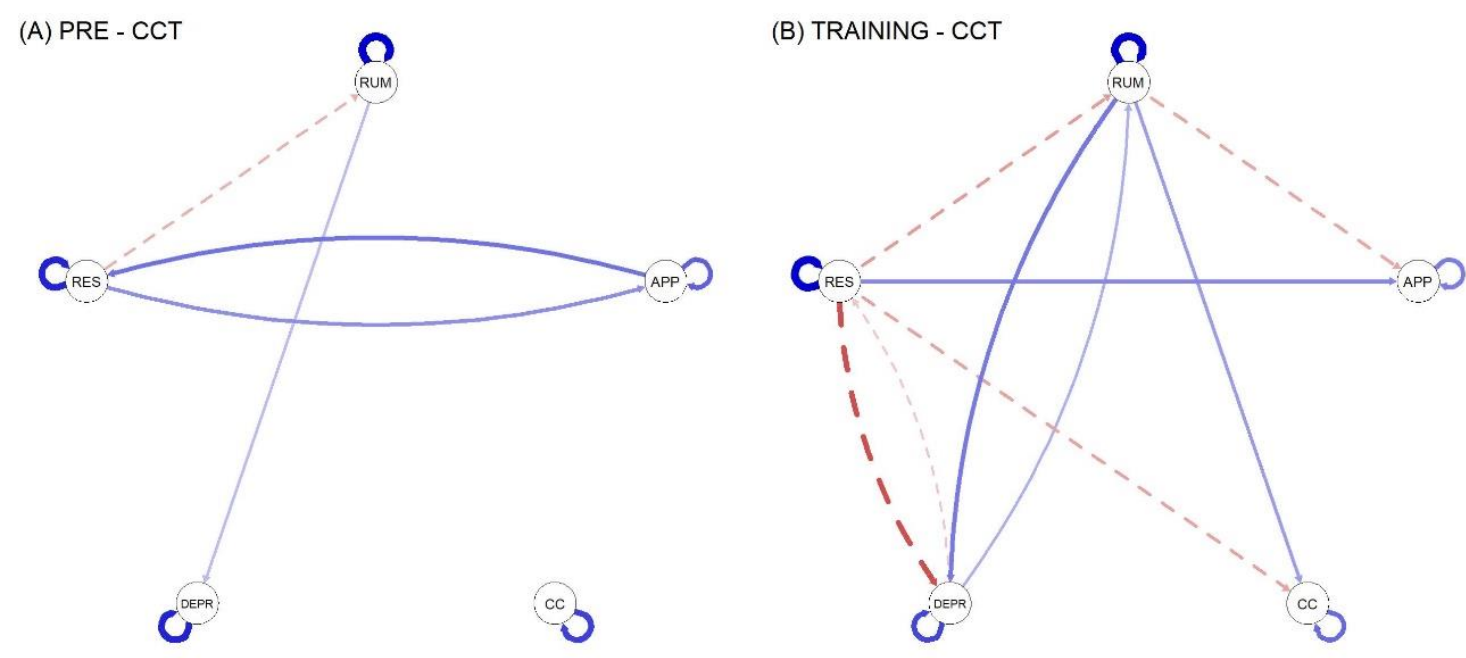

(C) POST - CCT

o

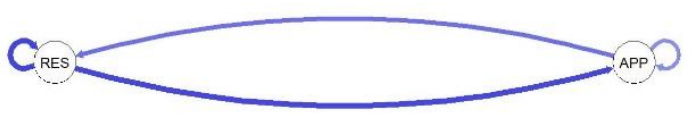

(D) PRE - ACT

(E) TRAINING - ACT

$\Omega$

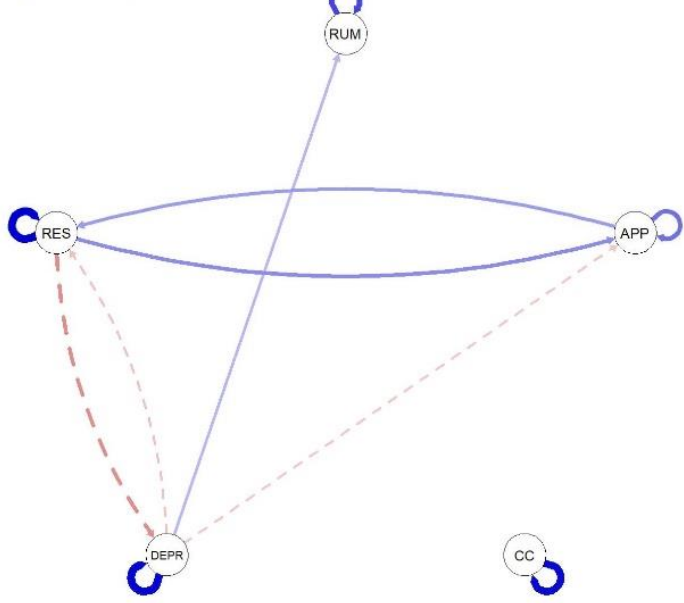

(F) POST - ACT

0

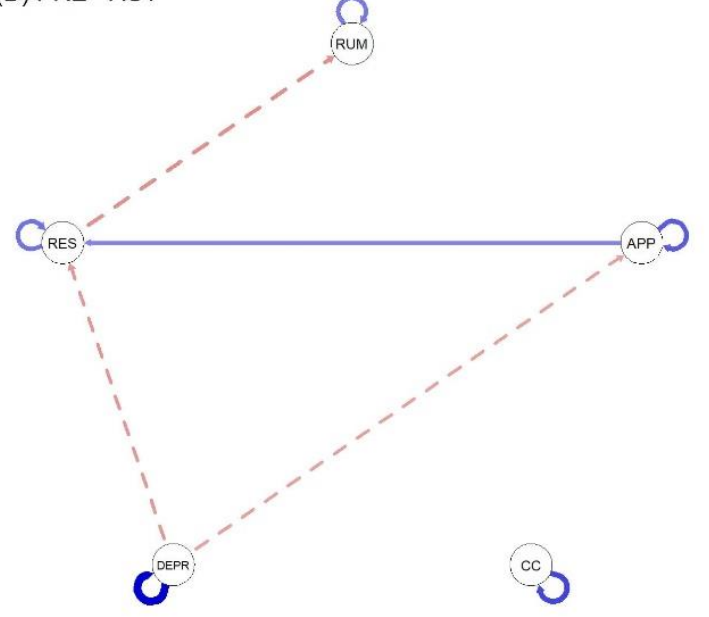

$\stackrel{\infty}{0}$

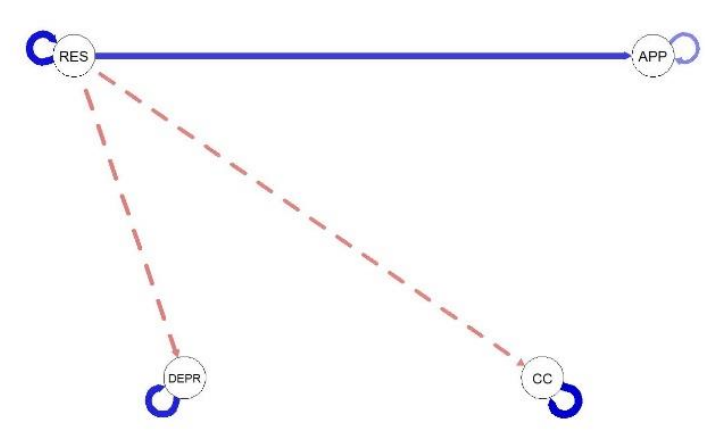

Figure 4. Effects of Cognitive Control Training on the temporal networks 
Note: $\mathrm{CCT}=\mathrm{Cognitive}$ Control Training; $\mathrm{ACT}=$ Active Control Training; RUM=Rumination, APP=Positive Appraisal, DEPR=Depressive symptomatology, $\mathrm{CC}=$ Cognitive Complaints, $\mathrm{RES}=$ Resilience 
(A) PRE - CCT

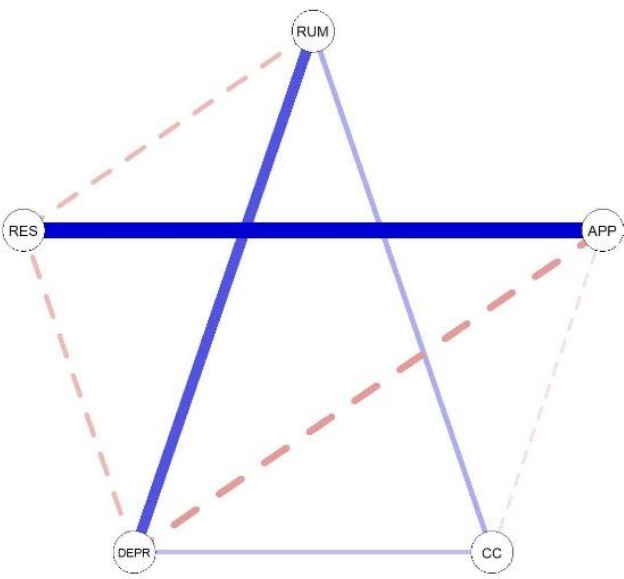

(D) PRE - ACT

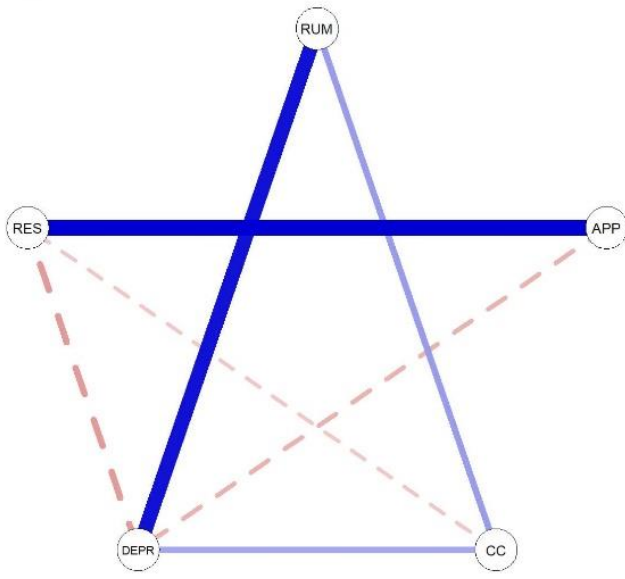

(B) TRAINING - CCT

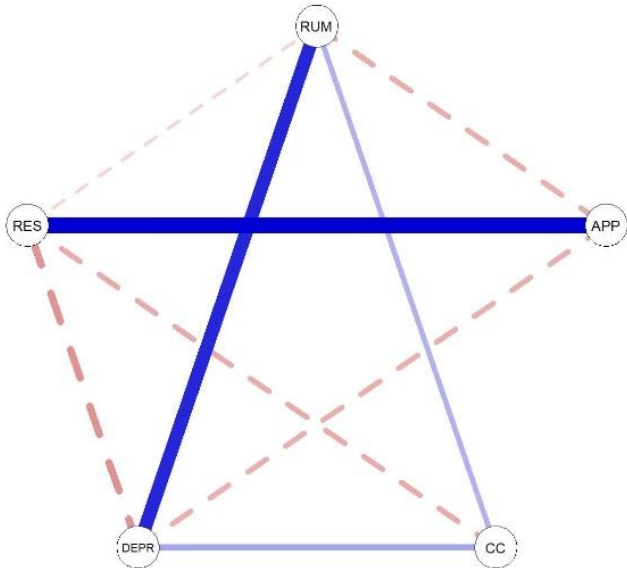

(E) TRAINING - ACT

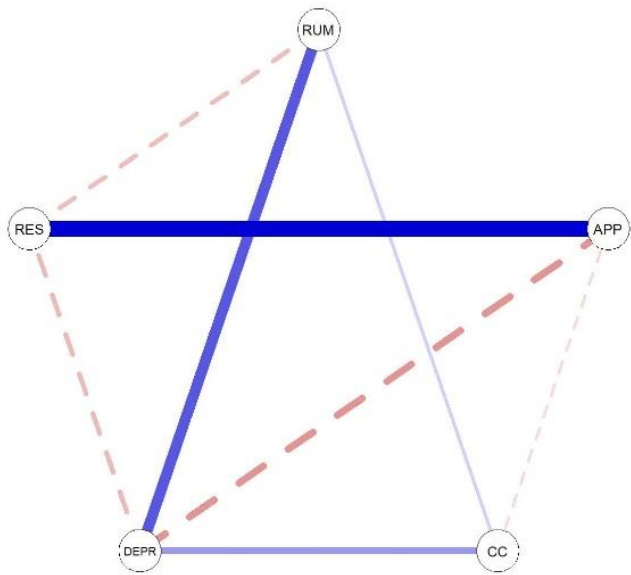

(C) POST - CCT

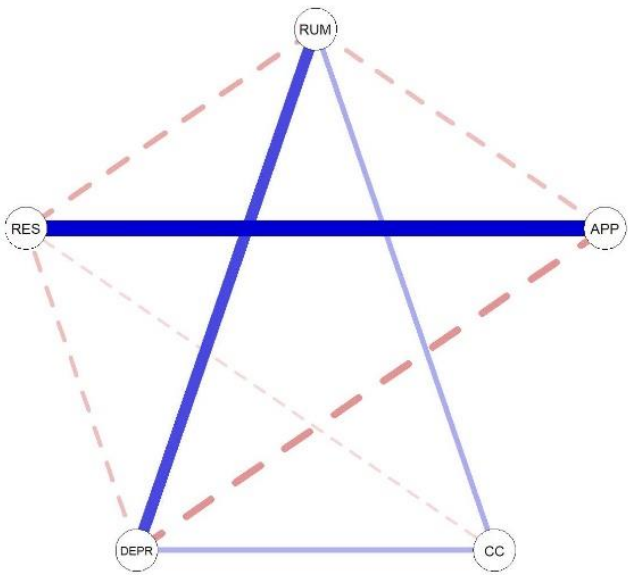

(F) POST - ACT

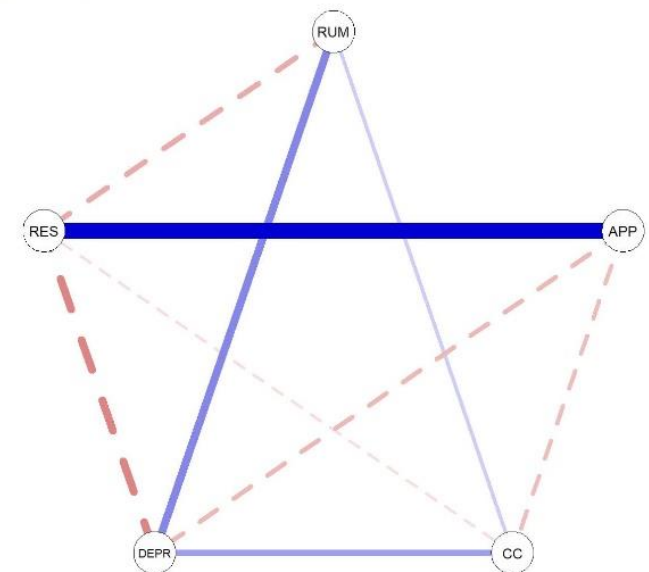

Figure 5. Effects of Cognitive Control Training on the contemporaneous networks

Note: $\mathrm{CCT}=$ Cognitive Control Training; ACT=Active Control Training; RUM=Rumination, APP=Positive Appraisal, DEPR=Depressive symptomatology, $\mathrm{CC}=$ Cognitive Complaints, $\mathrm{RES}=$ Resilience 